\title{
Executive Control Over Cognition: Stronger and Earlier Rule-Based Modulation of Spatial Category Signals in Prefrontal Cortex Relative to Parietal Cortex
}

\author{
Shikha J. Goodwin, ${ }^{1,5}$ Rachael K. Blackman, ${ }^{3,4,5}$ Sofia Sakellaridi, ${ }^{2,5}$ and Matthew V. Chafee ${ }^{2,3,5}$ \\ ${ }^{1}$ Department of Biomedical Engineering and ${ }^{2}$ Center for Cognitive Sciences, University of Minnesota, ${ }^{3}$ Department of Neuroscience and ${ }^{4} \mathrm{MD} / \mathrm{PhD}$ Training \\ Program, University of Minnesota Medical School, Minneapolis, Minnesota 55455, and ${ }^{5}$ Brain Sciences Center, Veterans Affairs Medical Center, \\ Minneapolis, Minnesota 55417
}

Human cognition is characterized by flexibility, the ability to select not only which action but which cognitive process to engage to best achieve the current behavioral objective. The ability to tailor information processing in the brain to rules, goals, or context is typically referred to as executive control, and although there is consensus that prefrontal cortex is importantly involved, at present we have an incomplete understanding of how computational flexibility is implemented at the level of prefrontal neurons and networks. To better understand the neural mechanisms of computational flexibility, we simultaneously recorded the electrical activity of groups of single neurons within prefrontal and posterior parietal cortex of monkeys performing a task that required executive control of spatial cognitive processing. In this task, monkeys applied different spatial categorization rules to reassign the same set of visual stimuli to alternative categories on a trial-by-trial basis. We found that single neurons were activated to represent spatially defined categories in a manner that was rule dependent, providing a physiological signature of a cognitive process that was implemented under executive control. We found also that neural signals coding rule-dependent categories were distributed between the parietal and prefrontal cortex- however, not equally. Rule-dependent category signals were stronger, more powerfully modulated by the rule, and earlier to emerge in prefrontal cortex relative to parietal cortex. This suggests that prefrontal cortex may initiate the switch in neural representation at a network level that is important for computational flexibility.

\section{Introduction}

Human cognition is only partly dictated by incoming sensory information at each moment, proceeding along a course that is also determined by internal state variables, such as rules, goals, or objectives. The capacity to modify computation according to internal context is often referred to as executive control, and while existing evidence indicates that prefrontal cortex is essential for this capability in both monkeys and humans (Goldman-Rakic, 1987; Wallis et al., 2001; Miller et al., 2002; Nakahara et al., 2002; Genovesio et al., 2005; Mansouri et al., 2006; Stoet and Snyder, 2009), we have an incomplete understanding of the neural mech-

\footnotetext{
Received July 13, 2011; revised Dec. 1, 2011; accepted Dec. 21, 2011

Author contributions:S.J.G. and M.V.C. designed research; S.J.G., R.K.B., and S.S. performed research;S.J.G., S.S., and M.V.C. analyzed data; S.J.G., R.K.B., S.S., and M.V.C. wrote the paper.

This work was supported by United States Public Health Service, National Institutes of Health Grants R01MH077779 and R24MH069675, the Department of Veterans Affairs, and the American Legion Brain Sciences Chair. We gratefully acknowledge Apostolos Georgopoulos for his support of this work, as well as important insights regarding the study and this manuscript. We thank David Crowe for valuable discussions regarding the data analysis and this manuscript. We thank Angus MacDonald and Scott Sponheim for discussions regarding the experimental design of the behavioral paradigm and its potential translation to studies of human cognition. We thank Dean Evans for excellent surgical assistance, animal care, and technical support. We thank Dale Boeff for expert computer programming and technical support. The views and opinions expressed in this work are those of the authors solely and not those of the United States Federal Government.

Correspondence should be addressed to Matthew V. Chafee, Brain Sciences Center (11B), Veterans Affairs Medical Center, 1 Veterans Drive, Minneapolis, MN 55417. E-mail: chafe001@umn.edu.

DOI:10.1523/JNEUROSCI.3585-11.2012

Copyright $\odot 2012$ the authors $\quad 0270-6474 / 12 / 323499-17 \$ 15.00 / 0$
}

anisms that exert executive control over cognitive processing at the level of prefrontal neurons and networks.

To characterize the neural mechanisms involved in computational flexibility and executive control over spatial cognitive processing at the network level, we simultaneously recorded neural activity in prefrontal and parietal cortex of monkeys as they assigned the same set of visual stimuli to alternative spatial categories on the basis of alternative categorization rules (Ashby and Maddox, 2005). In our task, monkeys applied different criteria to flexibly regroup the same set of stimulus positions into alternative spatial categories, placing categorization as a cognitive process under executive control. (Spatial categories each consisted of a set of spatial positions grouped together by virtue of sharing a common spatial relationship to a visual stimulus serving as a category boundary.) Based on evidence that parietal neurons encode relative spatial position (Chafee et al., 2005, 2007; Crowe et al., 2008), we predicted that parietal neurons would encode spatial categories defined on the basis of relative position, and that the prefrontal-parietal network would be engaged to implement executive control over categorization when the spatial relationship that defined categories changed over trials.

In this experiment, we recorded from area $7 \mathrm{a}$ in the posterior parietal cortex and area 46 in the dorsolateral prefrontal cortexareas that share direct corticocortical projections (Cavada and Goldman-Rakic, 1989) and contain neurons that exhibit nearly identical patterns of activity during spatial cognitive tasks 

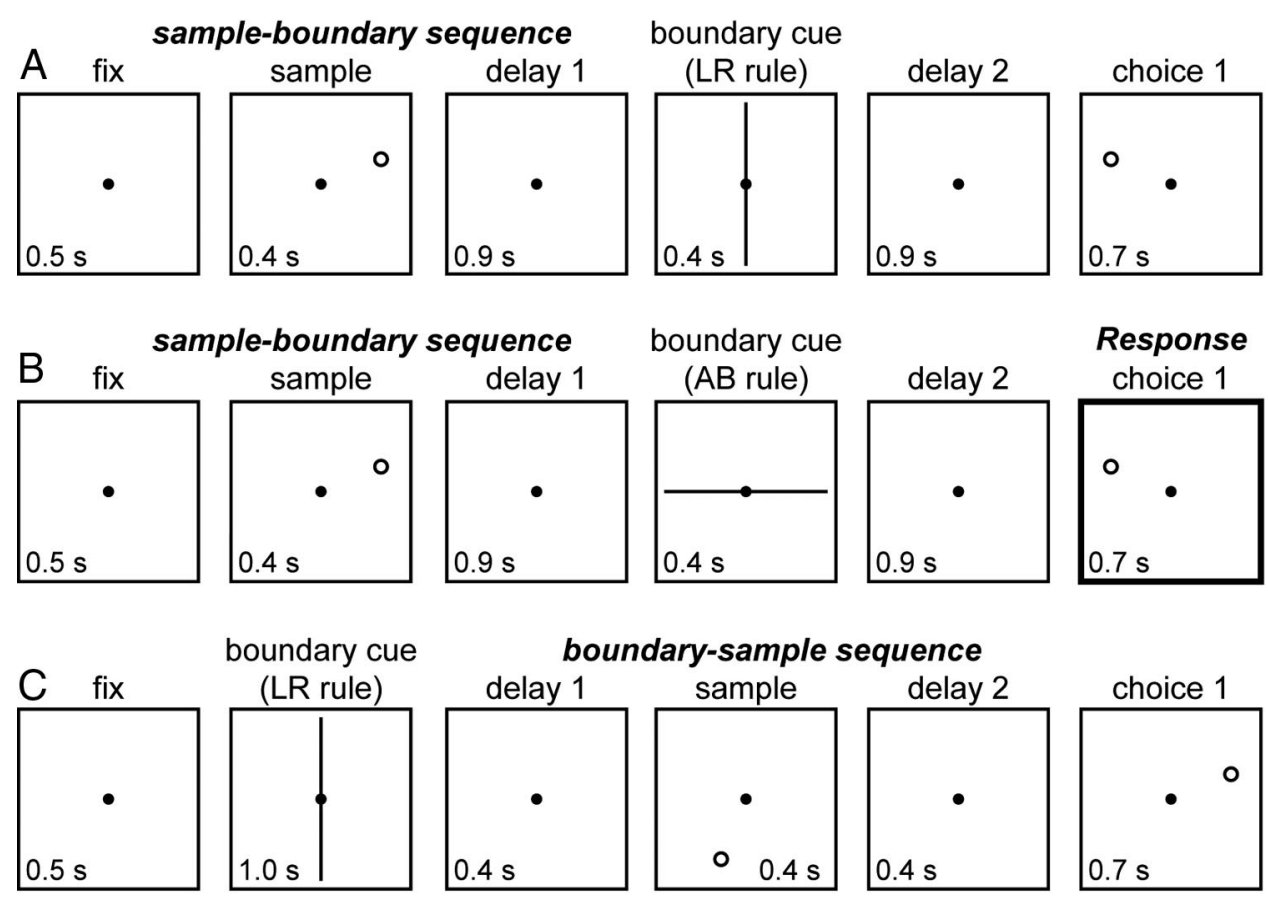

boundary cue
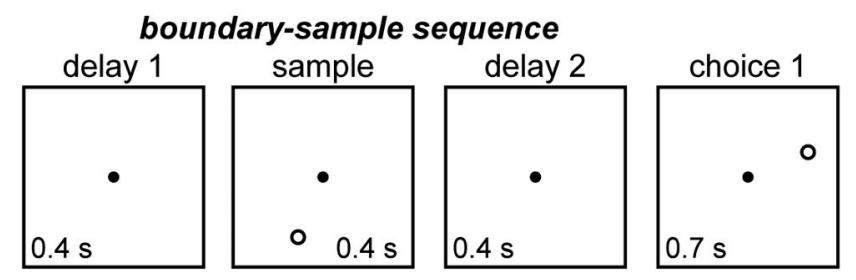

\section{Response}

choice 2
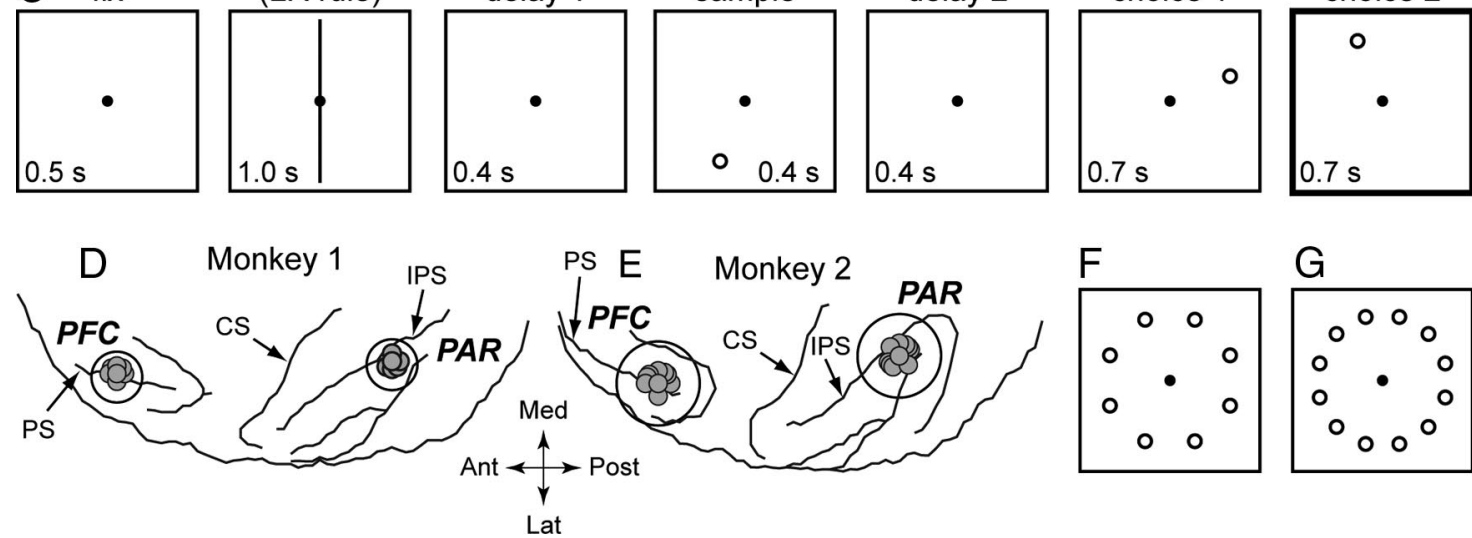

Figure 1. Event sequence of the DYSC task and locations of neural recording in parietal (PAR) and prefrontal (PFC) cortex. Each trial, we presented a small circular sample stimulus and a line serving as a boundary cue. We varied the order of presentation of these stimuli, using either a sample-boundary sequence or a boundary-sample sequence. $\boldsymbol{A}$, Categorizing stimuli according to the left/right (LR) rule under the sample- boundary sequence. Trials began with the presentation of a central gaze fixation target (gaze fixation was required throughout the trial until the response was made). A sample stimulus was presented at one of 8 or 12 randomly selected locations for $400 \mathrm{~ms}$, followed by a $900 \mathrm{~ms}$ delay period (delay 1). The LR rule was instructed by the presentation of the boundary cue in a vertical orientation for $400 \mathrm{~ms}$, followed by a $900 \mathrm{~ms}$ delay period (delay 2). The monkey was required to determine the spatial category of the sample position stored in working memory by evaluating its spatial relationship to the boundary cue. The sample on this trial belongs to the spatial category "right." After delay 2, two choice stimuli were sequentially presented for $700 \mathrm{~ms}$ each in random order, one in the opposite spatial category as the sample (choice 1; "left") and one in the same spatial category (choice 2; "right"). The DYSC task is a delayed category match-to-sample design. The monkey was rewarded (with a drop of juice) if it pressed the response key during the period of time that the matching choice was visible (located in the same spatial category as the sample; choice 2 on this trial). $\boldsymbol{B}$, Categorizing stimuli according to the above/below ( $A B$ ) rule under the sample- boundary sequence. The boundary cue is presented in a horizontal orientation instructing the AB rule on this trial, and choice 1 matches the spatial category of the sample ("above"). C, Categorizing stimuli according the LR rule under the boundary-sample sequence. In this case, the sample stimulus is assigned to a category based on a boundary cue stored in working memory. $\boldsymbol{D}, \boldsymbol{E}$, Locations of neural recordings in parietal and prefrontal cortex of monkeys 1 and 2 relative to positions of the principal sulcus (PS), central sulcus (CS), and intraparietal sulcus (IPS) as reconstructed from structural MRl images. The perspective is a top-down view of the left cerebral hemisphere. Anterior (Ant), posterior (Post), medial (Med), and lateral (Lat) directions are as indicated by the arrows. The larger open circles indicate inner diameter of recording chambers over parietal and prefrontal cortex. The smaller filled circles within each cortical area indicate regions sampled by electrode penetrations during neural recording. $\boldsymbol{F}$, Eight-position sample array. $\boldsymbol{G}$, Twelve-position sample array.

(Chafee and Goldman-Rakic, 1998, 2000). Neural signals associated with a variety of other cognitive processes are similarly distributed between prefrontal cortex and interconnected cortical areas (Freedman et al., 2003; Nieder and Miller, 2004; Muhammad et al., 2006), raising the question what unique contribution prefrontal cortex makes to distributed information processing in cortical networks. One possibility is that prefrontal cortex generates neural signals that become rapidly communicated to other cortical areas, in which case detecting the unique function of the prefrontal cortex at the single-neuron level might require finding which among several distributed signals are strongest or emerge first in prefrontal neurons. In the context of a rule-based categorization task, we found that neural signals representing the category of the stimulus as a function of the rule applied were stronger, earlier, and more powerfully modulated by the rule in prefrontal cortex relative to parietal cortex, suggesting that prefrontal cortex may lead in the executive control of the spatial cognitive processing required.

\section{Materials and Methods}

Animals. We trained two male macaque monkeys (5-8 kg) to perform the dynamic spatial categorization (DYSC) task (described below). After reaching a stable criterion level of performance (performing $>80 \%$ of trials correctly), the monkeys underwent aseptic surgery under gas anesthesia (isoflurane, 1-2\%) to place recording chambers (7 and $13 \mathrm{~mm}$ inner diameter in monkeys 1 and 2, respectively) above craniotomies overlying the dorsolateral prefrontal cortex and posterior parietal cortex in the left cerebral hemisphere, as well as to fix titanium screws and posts to the skull to restrain head position as necessary for neural recording. The prefrontal chamber was centered over Brodmann's area 46 in the principal sulcus. The parietal chamber was centered over area $7 \mathrm{a}$ in the inferior parietal lobule (Fig. 1D,E). We 
administered analgesia for several days postoperatively (Buprenex; 0.05 $\mathrm{mg} / \mathrm{kg}$ bid, i.m.). After monkeys recovered from surgery, we started neural recording during DYSC task performance. All surgical and animal care procedures conformed to the Principles of Laboratory Animal Care of the NIH and protocols approved by the Animal Care and Use Committees of the Minneapolis Veterans Affairs Medical Center and the University of Minnesota.

Dynamic spatial categorization task. The DYSC task (Fig. 1) required monkeys to determine whether a small circle (the sample stimulus) was located to one side of a line (the boundary cue) or the other, and to press a response key when a choice stimulus appeared on the same side of the boundary cue later in the trial. The boundary cue (Fig. $1 A$, boundary cue) constituted a category boundary that divided space into two regions, each containing a set of points grouped into a category by virtue of bearing the same spatial relationship to the boundary. (For example when the boundary cue was vertical, all points to the left of the boundary constituted one spatial category; all points to the right another.)

Each trial, we presented first a sample stimulus followed after a delay by the category boundary (Fig. $1 A, B$, sample-boundary sequence), or we presented the two stimuli in reverse order, first the category boundary followed by the sample stimulus (Fig. 1C, boundary-sample sequence). Presenting the two stimuli at different times separated by an intervening delay ensured that the two stimuli were never simultaneously visible, and could not therefore jointly influence the receptive fields of visual neurons. Sample stimuli were small circles $\left(0.25-0.5^{\circ}\right.$ diameter; either white or yellow), and boundary cues were blue lines ( 13 or $21.5^{\circ}$ in length), backprojected by an LCD projector (NEC MT820) onto an otherwise dark translucent screen located $65 \mathrm{~cm}$ in front of the monkey. The location of the sample was selected randomly from a circular array of either 8 or 12 positions located on an imaginary circle at an eccentricity of $13^{\circ}$ from the fixation target (Fig. $1 F, G$; monkey 1 performed the task with both 8 - and 12-position arrays; monkey 2 performed the task with the 8 -position array). We located sample positions in the arrays so that they did not fall on the boundary cue when presented in either the vertical (Fig. $1 \mathrm{~A}$, boundary cue) or horizontal (Fig. $1 \mathrm{~B}$, boundary cue) orientations (to ensure that each position in the sample array had a defined spatial category for both boundary orientations).

Monkeys initiated the trial by directing their gaze straight ahead toward a fixation target $\left(0.25^{\circ}\right.$ red circle $)$ presented directly in front of them (Fig. $1 A-C$, fix). If the monkey's gaze deviated by $>2.5^{\circ}$ from the fixation target at any point during the trial, the trial was terminated without reward. After $500 \mathrm{~ms}$ of central fixation, we presented the first visual stimulus in the sequence, either the sample stimulus (Fig. $1 A, B$, sample), or the boundary cue (Fig. $1 C$, boundary cue). We presented the boundary cue either in a vertical (Fig. $1 A, C$ ) or horizontal (Fig. $1 B$ ) orientation each trial (always centered on and passing through the gaze fixation target). After the disappearance of the first stimulus in the sequence, a delay period of 400 or $900 \mathrm{~ms}$ followed (Fig. $1 A-C$; delay 1 ). At the end of delay 1, the second stimulus was presented for $400 \mathrm{~ms}$ - either the boundary cue (Fig. $1 A, B$ ) or the sample stimulus (Fig. 1C), depending on the stimulus sequence used. An important aspect of the task design was that the rule-dependent category of the sample stimulus was only defined after the second stimulus was presented in either stimulus sequence. After the offset of the second stimulus, a second delay period followed (Fig. 1A-C; delay 2; 400 or $900 \mathrm{~ms}$ ). At the end of delay 2, we presented two choice stimuli (small circles) one at a time for $700 \mathrm{~ms}$ each in random order. One choice was always a match (located on the same side of the boundary cue and therefore belonging to the same spatial category as the sample), and the other choice was always a nonmatch (located on the other side of the boundary cue and belonging to the opposite spatial category). If the monkeys pressed the response key during the period of time that that matching choice stimulus was visible (Fig. $1 A-C$; response; boxes with thicker outlines), we terminated the trial and delivered a liquid reward ( $0.1-0.2 \mathrm{ml}$ of sweetened water). If the monkey pressed the response key when the nonmatch choice was visible, we terminated the trial without reward. The direction of the required motor response did not vary over trials, so that neural signals varying with the spatial features of the task were not likely to reflect motor plans of varying direction. Furthermore, the timing of the motor response was unpredict- able in advance of the choice sequence because the order of choices (match and nonmatch) was randomized over trials. Monkeys performed sets of trials consisting of 12 or 15 repetitions of each sample position under each of the two boundary orientations.

On trials in which the boundary cue was vertical (Fig. $1 A, C$, boundary cue), it instructed the LR (left/right) categorization rule, and monkeys were required to determine whether the sample stimulus was located to the left or right of the category boundary. On trials in which the category boundary was horizontal (Fig. $1 B$, boundary cue), it instructed the $\mathrm{AB}$ (above/below) rule, and monkeys were required to determine whether the sample stimulus was located above or below the category boundary. Changing the boundary orientation over trials therefore required reclassifying the circular stimulus array into orthogonal sets of spatial categories. The boundary cue served to instruct a categorization rule in the sense that its orientation dictated which of two independently varying dimensions of the sample stimulus (horizontal or vertical position) was relevant to category membership and which grouping criterion to apply to that dimension to determine the spatial category. When the boundary cue was vertical, the position of the sample along the horizontal axis was relevant to category membership and its vertical position was irrelevant. When the boundary cue was horizontal, the converse was true. We varied boundary orientation either randomly over trials, or in blocks of random length (between 7 and 12 trials).

Choice stimuli were placed at the same eccentricity as sample stimuli $\left(13^{\circ}\right.$ from the fixation target). For the boundary-sample data in monkey 1 , we selected match and nonmatch choice positions from the same stimulus array used to position samples (Fig. $1 F, G$ ), with the constraint that the two choices on each trial were located at equal distances from the active boundary. For the remaining data (the sample-boundary data in monkeys 1 and 2), we more fully randomized the position of choice stimuli, placing them at randomly selected angles on either side of the active category boundary, with the constraint that match and nonmatch choices fell at equal distances from the boundary, and the angles of choice stimuli were $>30^{\circ}$ from the active category boundary. For example, to select a position for a choice stimulus to the left of the LR boundary, we selected an angle at random between 120 and $240^{\circ}$. We then placed the right choice on the opposite side of the boundary, at one of the two angles (again selected at random) that would place the right choice at the same distance from the category boundary as the left choice. Therefore, it was not possible to predict the location of one choice from the position of the other with certainty.

Neural recording. We recorded neural activity in prefrontal and posterior parietal cortex simultaneously using two 16 electrode Eckhorn microdrives (Thomas Recording). Electrodes were $70-\mu \mathrm{m}$-outer diameter glass-coated platinum iridium fibers (impedance, $1-2 \mathrm{M} \Omega$ ), advanced independently under computer control into the brain. The electrical signal from each electrode was amplified (gain of 2500) and bandpass filtered (cutoff frequencies of $0.5 \mathrm{~Hz}$ and $5 \mathrm{kHz}$ ). The waveforms of the action potentials of individual neurons were isolated on-line using a combination of time-amplitude window discriminators (Bak Electronics) and waveform discriminators (Alpha Omega Engineering). The timing of spike occurrence was stored to disk with $40 \mu$ s resolution (DAP 5200a Data Acquisition Processor; Microstar Laboratories). We typically isolated the action potentials of 15-25 parietal neurons and 15-25 prefrontal neurons concurrently, and recorded the activity of these neuronal ensembles as monkeys performed the DYSC task

Data analysis. We applied ANOVA and linear regression to evaluate the influence of sample position, rule, and spatial category on the firing rate of single neurons. We in addition used pattern classification analysis to decode sample position, rule, and spatial category from patterns of population activity in parietal and prefrontal cortex. We performed the regression and decoding analyses using neural firing rates measured in successive time bins. This produced a time course of regression coefficients or decoding accuracy that allowed us to quantify variation in the representation of position, rule, and category by both single neurons and populations in parietal and prefrontal cortex.

ANOVA/ANCOVA. We used different analyses to isolate neural signals coding position, rule, and category on sample-boundary and boundary-sample trials (different approaches were required to isolate 
position and category signals depending on the order in which sample and boundary cue were presented).

In the sample-boundary data, we presented the sample stimulus first. The spatial category of the sample was defined later in the trial when the boundary cue appeared (because the category was a joint function of stimulus position and boundary orientation). To identify neural activity that varied as a function of sample position (the first visual stimulus presented in this data set), we performed a one-way ANOVA on firing rates in the sample stimulus and subsequent delay period using sample position as the single factor. To identify neural activity that varied as a function of spatial category and rule (both defined when the second stimulus, the boundary cue, appeared), we performed a three-way ANCOVA on firing rates during the boundary cue and subsequent delay period. The three factors in this analysis were boundary orientation (LR or $\mathrm{AB}$ ), horizontal sample category (left or right), and vertical sample category (above or below). We defined both the horizontal and vertical category of the sample stimulus based on its location within the left, right, upper, or lower halves of the circular stimulus array (regardless of the orientation of the boundary cue). To factor out a potential influence of the spatial location of the preceding sample stimulus on neural activity, we included the firing rate of each neuron during the preceding sample and delay periods (when spatial position was defined but category was not) as a covariate in the analysis. Neural signals significantly influenced by the interaction between rule and category reflected rule-dependent category-selective activity and were of particular interest for their relevance to executive control of cognitive processing.

On boundary-sample trials, we presented the boundary cue instructing the categorization rule first, followed by the sample stimulus. The spatial position and spatial category of the sample were therefore simultaneously defined by the presentation of the sample stimulus. To identify neural activity that varied as a function of the boundary cue, we performed a one-way ANOVA on firing rates in the boundary cue and following delay periods using the orientation of the boundary cue as the single factor. We performed two separate ANOVAs on firing rate measured during the sample stimulus and subsequent delay period that tested for a significant influence of horizontal and vertical categories, respectively. Each analysis was a three-way design in which the three factors were rule, category, and within-category position. In the analysis evaluating the influence of horizontal category, the category factor had two levels (left and right), and the within-category position factor had four or six levels corresponding to the vertical position of the sample in the 8-or 12-sample position arrays (Fig. $1 F, G$ ). In the analysis evaluating the influence of vertical category, the category factor had two levels (above or below), and the within-category position factor had again four or six levels reflecting the horizontal position of the sample depending on the sample array used. We defined category-selective neurons in this dataset as those having firing rates that varied significantly across but not within spatial category, in either the horizontal or vertical analyses. Ruledependent category neurons were those in which the interaction between rule (boundary orientation) and category was additionally significant. Neurons coding sample position were identified as neurons with activity varying as a function of within-category position that did not meet the above criteria for category selectivity.

Decoding analysis. We also performed a pattern classification analysis (Klecka, 1980; Johnson and Wichern, 1998; Crowe et al., 2010) to decode task-defined behavioral variables from patterns of neural activity in parietal and prefrontal cortex. To obtain a measure of physiological signal strength and timing that was representative of the cortical areas of interest, we performed the decoding analysis at the population level aggregating neurons over recording sessions. We matched the activity of neurons recorded at different times based on the trial repetition of each stimulus condition. We then used the pattern of firing rates over the neurons in the population on each trial repetition to decode the position of the sample stimulus, the orientation of the boundary cue, the spatial category of the sample, and the rule-dependent spatial category of the sample. We first performed the ANOVA/ANCOVA on the activity of single neurons as described above, and then ranked neurons according to the significance of the decoded variable on firing rate. We then compared decoding results when using the most significant 70 neurons, the most significant
200 neurons, or all significant neurons $(p<0.05)$ in parietal and prefrontal cortex to decode each task variable. Comparing decoding accuracy across cortical areas using all significant neurons evaluated differences in neural representation that could potentially involve differences in the numbers of recruited neurons. Comparing decoding accuracy across cortical areas using equal numbers of the most significant neurons held the size of the populations compared constant [removing the number of neurons as a potential factor responsible for differences in decoding accuracy (Averbeck et al., 2003)]. This evaluated differences in neural representation based on the neurons with the strongest signals in each cortical area.

We performed each decoding analysis in a time-resolved manner, using firing rates measured within a sliding window of three or five consecutive $50 \mathrm{~ms}$ time bins, advanced in $50 \mathrm{~ms}$ steps throughout the trial. At each time step, we measured the firing rate of each neuron within the short sequence of three or five $50 \mathrm{~ms}$ time bins, and concatenated these rate measurements over neurons in the population to yield a population rate vector that captured the distribution of firing rates over the population during a short span of time (150-250 ms).

We used fivefold cross-validation in the decoding analysis so that the neural data we used to decode each behavioral variable never directly contributed to the parameters of the classification functions we applied. This prevented overfitting the classification functions to noise in the sample data and helped to ensure that the classification was robust. We trained the classifier using neural activity on four-fifths of the trials, and then decoded behavioral variables using neural activity on the remaining one-fifth of trials, iterating the analysis (classifying successive one-fifth of the trials) until all trials were classified.

Each trial repetition in the decoding analysis was represented by a vector of firing rates capturing the activity pattern in the population. Training the classifier consisted of computing the mean population activity vector associated with each level of the decoded variable in the training data (defining the population activity patterns on left and right trials, for example, when decoding horizontal category), as well as computing the covariance matrix between neurons. We then computed the Euclidean distance between the population activity vector on each test trial and the various mean activity vectors derived from the training data, and converted these distances to posterior probabilities under the assumption that the distribution of vectors in each group was multivariate normal. We classified each test trial to the value of the decoded variable associated with the greatest posterior probability, using the "classify" function in the MATLAB statistical toolbox (MathWorks) to compute the posterior probabilities. The proportion of trials in which the decoding analysis returned the correct value of the behavioral variable given the stimuli displayed each trial, based only on the activity patterns observed, provided a metric that quantified the strength with which population activity coded each behavioral variable. We decoded horizontal categories (left and right) using the subset of trials performed under the LR rule, and we decoded vertical categories (above and below) using the subset of trials performed under the $\mathrm{AB}$ rule. We then averaged the results obtained when decoding horizontal and vertical categories.

Decoding rule-dependent categories on error trials. To evaluate whether neural signals coding rule-dependent category related to successful performance, we compared the accuracy of decoding on correct and error trials. We restricted the populations used to neurons that had been recorded in experiments containing a minimum of 30 errors (this provided an acceptable trade-off between the numbers of neurons available and the numbers of error trials analyzed). We then ranked neurons according the significance of the influence of the interaction between rule and category on firing rate, and selected the top 50 neurons in each cortical area and monkey (one-half with preferences for horizontal categories, one-half with preferences for vertical categories) to include in the population. To maximize the sensitivity of the analysis to differences in signal strength between correct and error trials, we measured firing rates in a single, larger time bin starting with the onset of the second stimulus in the sequence (boundary or sample), when rule-dependent category was defined, and ending with the onset of the first choice stimulus. We then decoded rule-dependent spatial category as a single variable with four levels (left, right, above, and below) reflecting the combination of 


\section{MONKEY 1}
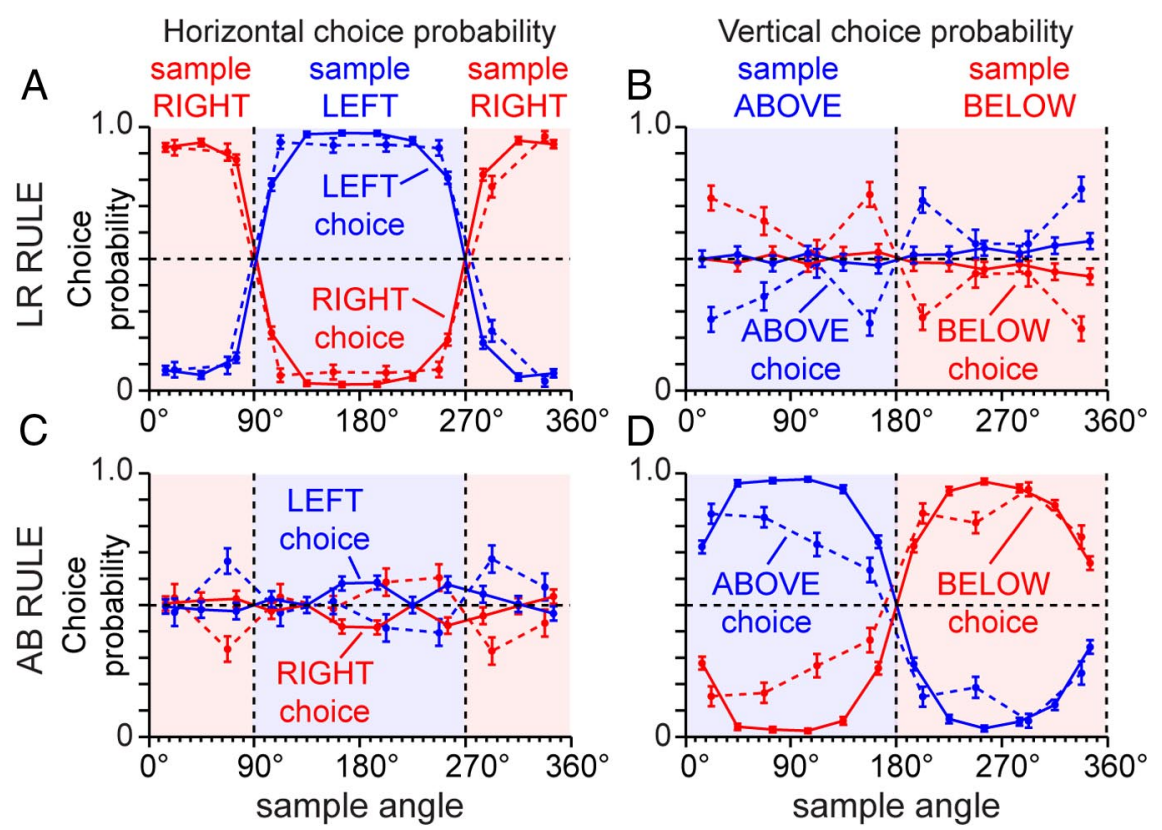

MONKEY 2
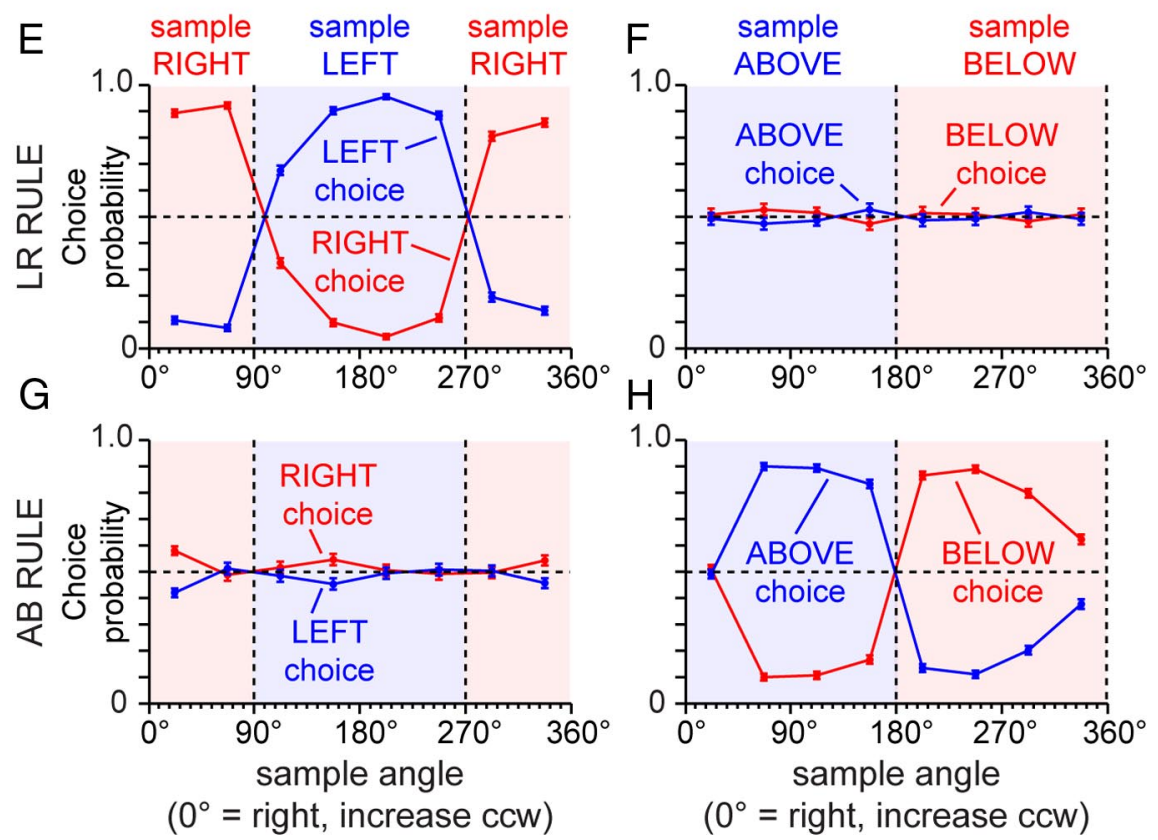

Figure 2. Behavioral performance of monkeys $1(\boldsymbol{A}-\boldsymbol{D})$ and $2(\boldsymbol{E}-\boldsymbol{H})$ in the DYSC task. The horizontal axis of each plot indicates the position of the sample stimulus, in degrees angle counterclockwise from the direction to the right of the fixation target (defined as $0^{\circ}$; sample eccentricity was fixed at $13^{\circ}$ ). The red-and blue-shaded regions indicate spans of sample angle corresponding to the spatial categories right and left $(\boldsymbol{A}, \boldsymbol{C}, \boldsymbol{E}, \boldsymbol{G})$, above and below $(\boldsymbol{B}, \boldsymbol{D}, \boldsymbol{F}, \boldsymbol{H})$, respectively. The lines and symbols indicate the probability (proportion of trials) that monkeys selected choices that were located in the right (red lines) or left (blue lines), above (blue lines) or below (red lines) spatial categories for each sample position and rule. The vertical dashed lines indicate angles corresponding to the category boundary under a given rule. $\boldsymbol{A}, \boldsymbol{B}$, Performance of monkey 1 under the $L$ R rule, when the boundary cue was vertical. The solid lines indicated performance on trials using the boundary-sample sequence, and the dashed lines performance on trials using the sample-boundary sequence. $\boldsymbol{A}$, Under the LR rule, monkey 1 selected left choices with high probability (blue symbols and lines) when the sample category was left (blue shading), and right choices with high probability (red symbols and lines) when the sample category was right (red shading). $\boldsymbol{B}$, Under the LR rule, the vertical category of the choices selected by monkey 1 (above or below) did not relate systematically to the vertical category of the sample. $C, D$, Performance of monkey 1 under the $A B$ rule, when the boundary cue was horizontal. $C$, Under the AB rule, the horizontal category of the choices selected by monkey 1 (left or right) did not relate systematically to the horizontal category of the sample. $\boldsymbol{D}$, In contrast, under the $A B$ rule, monkey 1 selected above choices with high probability (blue symbols and lines) when the sample category was above (blue shading), and below choices with high probability (red symbols and lines) when the sample category was below (red shading). $\boldsymbol{E}-\boldsymbol{H}$, Corresponding data for monkey 2. sample position and boundary orientation. We trained the classifier using population activity patterns on correct trials, and then decoded rule-dependent category on error trials.

Category selectivity index. We computed a category-selectivity index (Freedman et al., 2001, 2002; Roy et al., 2010) for each neuron identified as category selective based on the results of the ANOVA/ANCOVA above. The index contrasts the mean difference in firing rate (during the second stimulus and subsequent delay period) for all pairs of sample positions located in different spatial categories (betweencategory difference, or BCD), and all pairs of sample positions located within the same spatial category (within-category difference, or WCD). The between-category and withincategory differences reflected the mean difference in firing rate for all pairs of sample positions oriented orthogonal and parallel to the category boundary, respectively. We then computed the category selectivity index for each neuron as the difference between these measures divided by their sum as follows: $(\mathrm{BCD}-\mathrm{WCD}) /(\mathrm{BCD}+\mathrm{WCD})$.

Sliding window linear regression analysis. We used a sliding window linear regression analysis to quantify the magnitude and timing of the effects that task variables exerted on the firing rates of single neurons. For this purpose, we measured the firing rate of each neuron in a $200 \mathrm{~ms}$ window advanced in $20 \mathrm{~ms}$ increments through the trial, and fit the firing rates observed at each time step to the following linear model: $F=\beta_{0}+\beta_{1} R+\beta_{2} H+\beta_{3} V+\beta_{4} R H+$ $\beta_{5} R V+\varepsilon$, where $F$ is firing rate, $R$ is the categorization rule signified by the orientation of the boundary cue ( $L R$ or $A B), H$ is the horizontal category of the sample (left or right), and $V$ is the vertical category of the sample (above or below). The $R H$ and $R V$ terms capture the interaction between the categorization rule and horizontal and vertical categories, respectively. We used the regstats function of the MATLAB statistical toolbox to estimate the regression coefficients $\beta_{0}-\beta_{5}$. The analysis produced a succession of regression coefficients for each neuron quantifying the magnitude of the relationship between firing rate and model parameters as the rate window was advanced through the trial.

Statistical tests of differences in neural signals between cortical areas. To test whether differences in decoding accuracy across areas were significant, we applied the $\mathrm{z}$ test of proportions to compare counts of trials producing accurate and erroneous decoding based on parietal and prefrontal activity in each time bin. We also used the sequential trials test (Armitage, 1975) to determine whether the time series of posterior probabilities computed by the decoding analysis and the time series of regression coefficients obtained in the regression analysis differed significantly between cortical areas. The sequential trials test computes upper and lower 95\% confidence boundaries around a mean zero accumulated difference between two time series, detecting a significant difference if the 


\section{Parietal}

\section{Position}
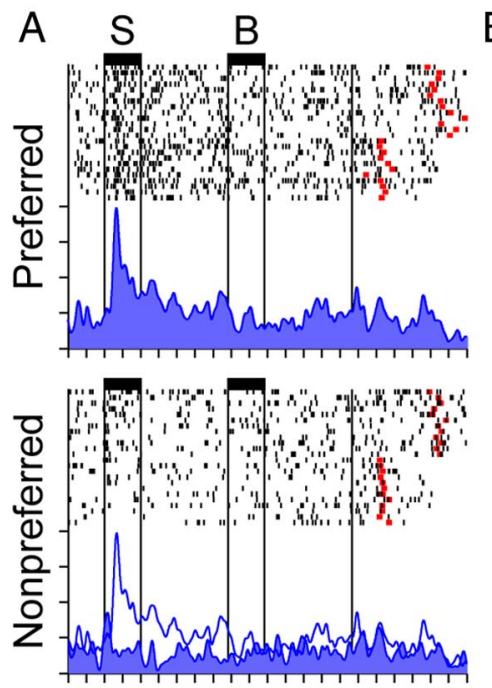

Rule
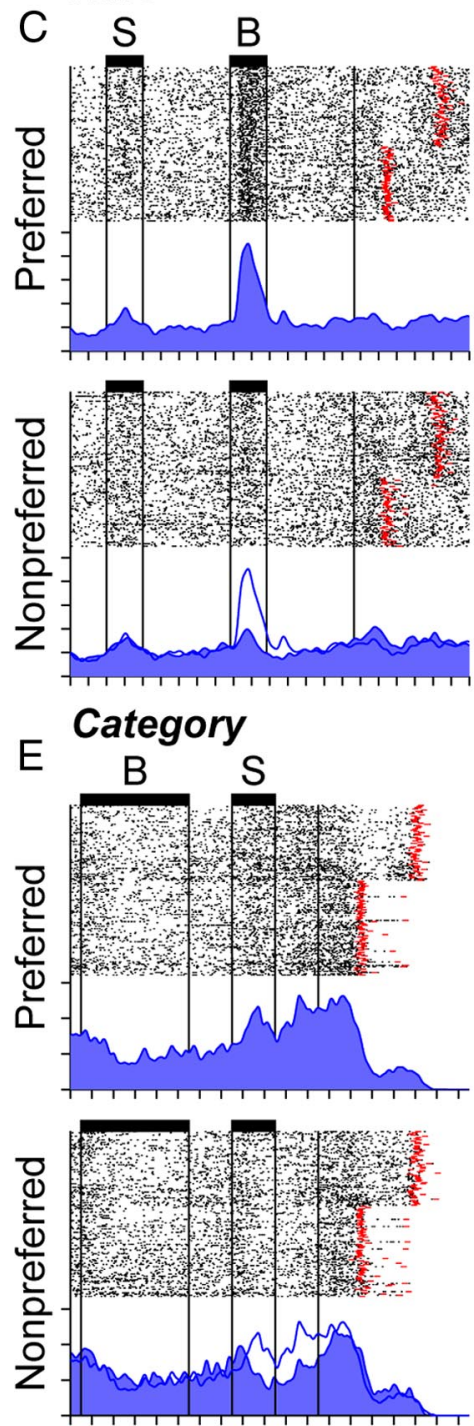

\section{Prefrontal}
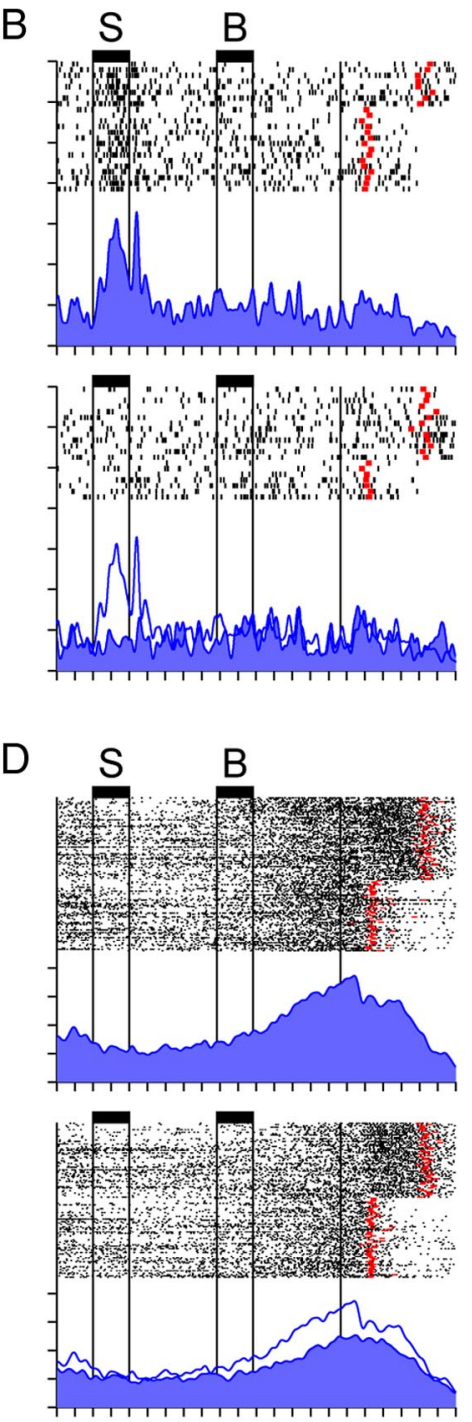

$\mathrm{F}$

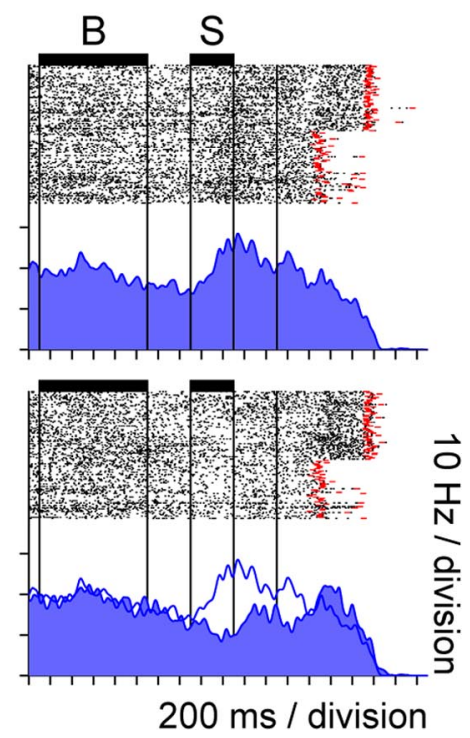

Figure 3. Rasters and spike density functions illustrate the activity of single neurons in parietal cortex $(\boldsymbol{A}, \boldsymbol{C}, \boldsymbol{E})$ and prefrontal cortex $(\boldsymbol{B}, \boldsymbol{D}, \boldsymbol{F})$ varying significantly $(p<0.05)$ as a function of sample position $(\boldsymbol{A}, \boldsymbol{B})$, rule (or boundary orientation) $(\boldsymbol{C}, \boldsymbol{D})$, and

accumulated difference crosses one of the boundaries. The distance between the upper and lower confidence boundaries increases over time to offset the increasing probability of getting larger accumulated differences by chance as a function of time after the start of the test. The slope of the confidence boundaries is a function of the $\alpha$ level (0.05) and the minimum effect size being evaluated. We implemented the analysis to evaluate significance using an effect size of 0.8 .

\section{Results}

\section{Behavioral performance}

Monkey 1 performed the DYSC task at a level of 86.5 and $84.9 \%$ correct for boundary-sample and sample-boundary stimulus sequences, respectively. Monkey 2 performed the DYSC task at a level of $85.0 \%$ correct for the boundary-sample stimulus sequence. The DYSC task required monkeys to flexibly parse the circular stimulus array into two orthogonal pairs of spatial categories: left/right and above/below. Figure 2 plots choice probability as a function of sample position and boundary orientation (sample position is indicated in polar coordinates along the $\mathrm{x}$-axis in degrees counterclockwise relative to $0^{\circ}$, to the right of fixation). The blue and red shading indicate spans of sample angle that correspond to left and right spatial categories (Fig. $2 A, C, E, G$ ), as well as above and below spatial categories (Fig. $2 B, D, F, H$ ).

Under the LR rule, instructed by the vertical boundary cue, both monkeys selected left choices with high probability (Fig. 2A,E, blue lines) when the sample had appeared in the left spatial category (Fig. 2A,E, blue-shaded regions), and right choices with high probability when the sample had appeared in the right spatial category (Fig. $2 A, E$, red lines and regions). The vertical category of the sample had little influence on vertical choice probability under the LR rule (Fig. $2 B, F$ ). On trials in which the category boundary was rotated to instruct the $\mathrm{AB}$ categorization rule (horizontal boundary), both monkeys no longer systematically based their choices on the horizontal category of the sample (Fig. $2 C, G$ ), but rather selected above choices with high probability (Fig. $2 \mathrm{D}, \mathrm{H}$, blue lines) when the sample had appeared in the above spatial category (Fig. $2 \mathrm{D}, \mathrm{H}$, blue-shaded regions), and be-

spatial category $(\boldsymbol{E}, \boldsymbol{F})$. Neural activity associated with the preferred position, rule, or category of each neuron is illustrated in the upper raster of each panel and activity on nonpreferred trials is illustrated in the lower raster (the thin blue line in the lower rasters shows activity on preferred trials for comparison). 

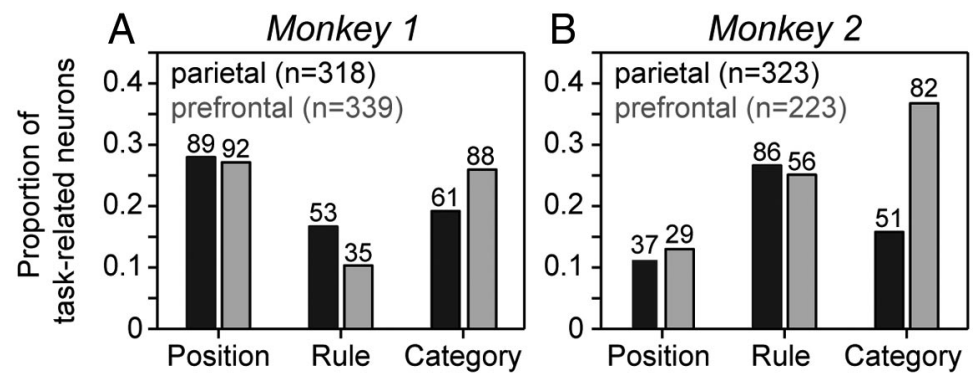

Figure 4. Proportion of task-related neurons in parietal cortex (black) and prefrontal cortex (gray) in which firing rate related significantly $(p<0.05)$ to the main effects of position, rule, and category in the ANOVA/ANCOVA (relative to the total number of neurons exhibiting any significant effect) in monkey $1(\boldsymbol{A})$ and monkey $2(\boldsymbol{B})$. Counts of parietal neurons (black) and prefrontal neurons (gray) exclude neurons significant for multiple main factors.

\section{MONKEY 1}
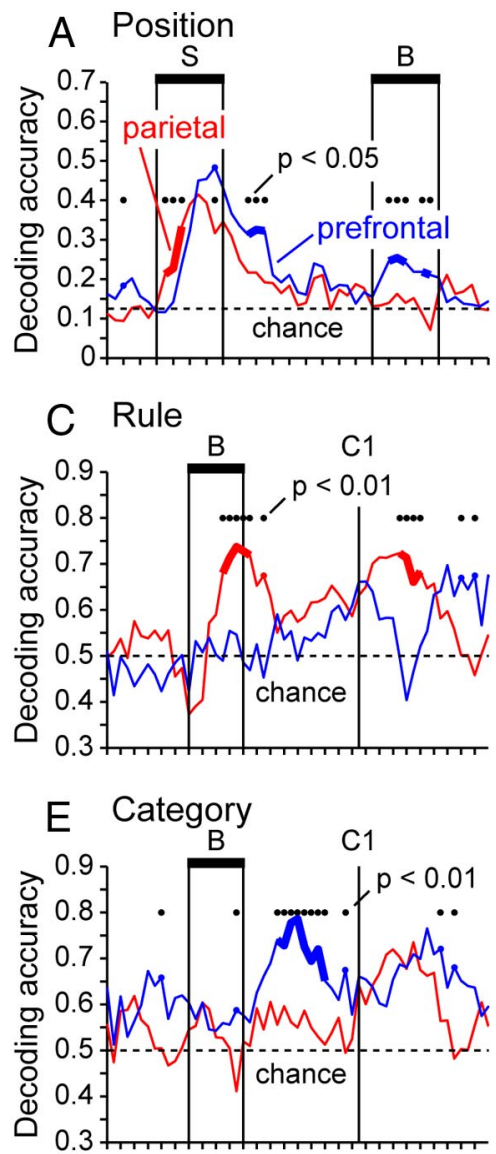

MONKEY 2
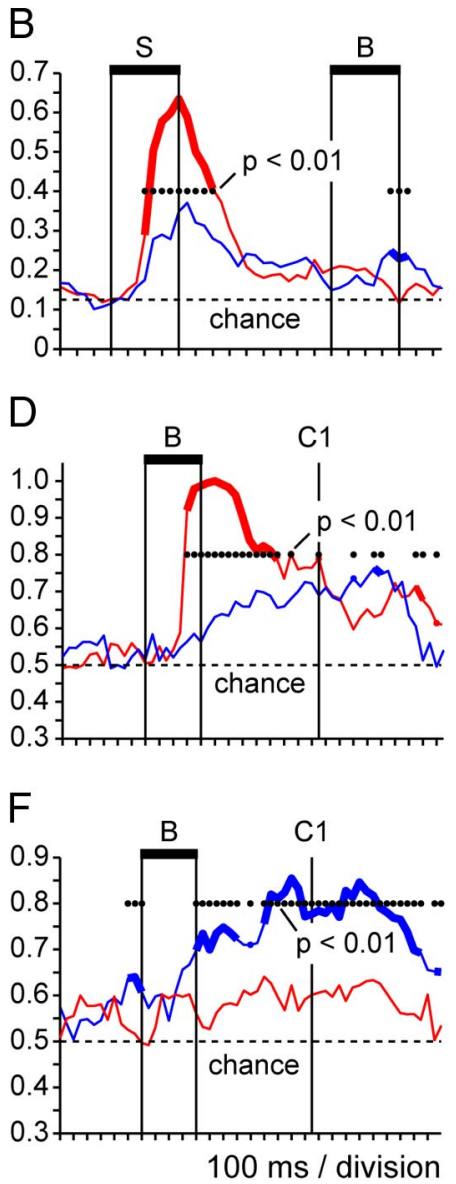

Figure 5. Plots of decoding accuracy over time indicate the proportion of trials in which sample position $(A, B)$, rule $(C, D)$, and spatial category $(\boldsymbol{D}, \boldsymbol{E})$ were accurately decoded from population activity patterns measured in successive $50 \mathrm{~ms}$ time bins in parietal cortex (red) and prefrontal cortex (blue). Data from monkey $1(\boldsymbol{A}, \boldsymbol{C}, \boldsymbol{E})$ and monkey $2(\boldsymbol{B}, \boldsymbol{D}, \boldsymbol{F})$ are plotted separately. Time bins in which the proportion of correctly decoded trials varied significantly between prefrontal and parietal cortex are indicated by filled circles and sections of the time courses plotted with a thicker line ( $z$ test of proportions, $\alpha$ level indicated in each panel).

low choices with high probability when the sample had appeared in the below spatial category (Fig. $2 D, H$, red lines and regions).

The performance of both monkeys was less accurate under the $A B$ rule, particularly on trials in which the sample stimulus had been presented near the horizontal category boundary (Fig. $2 \mathrm{D}, \mathrm{H}$; sample angles near 0 and $360^{\circ}$ ). This suggests that vertical categorization was more difficult for both monkeys. Nonetheless, the points of inflection of choice probability functions were lo- cated near the angles corresponding to the active category boundary, both for the LR rule (Fig. 2A,E; response probability functions cross near the vertical dashed lines indicating the angles of the vertical boundary cue at 90 and $270^{\circ}$ ) and also the $\mathrm{AB}$ rule (Fig. 2D, $\mathrm{H}$; functions cross near the vertical dashed lines indicating the angles of the horizontal boundary cue at 0 , 180 , and $360^{\circ}$ ). Furthermore, in both monkeys, choice probability clearly reflected an interaction between sample position and categorization rule, even for their weaker performance under the $\mathrm{AB}$ rule. These data are evidence that monkeys assigned the same set of visual stimuli to different sets of spatial categories in a flexible and rule-dependent manner.

\section{Neural database}

We recorded the activity of 1016 neurons in parietal cortex and 977 neurons in prefrontal cortex of two monkeys performing the DYSC task. In monkey 1, we recorded the activity of 504 parietal neurons and 496 prefrontal neurons. Of these, 705 neurons were recorded using the boundary-sample stimulus sequence and 295 neurons using the sample-boundary stimulus sequence. In monkey 2 , we recorded the activity of 512 parietal neurons and 481 prefrontal neurons using the sample-boundary stimulus sequence.

\section{Network representation of spatial position, rule, and category}

The position of the sample stimulus, the orientation of the boundary cue, and the spatial category of the sample comprised the primary spatial variables defined by the DYSC task. We found that populations of single neurons coding each of these spatial variables were distributed between parietal and prefrontal cortex (Fig. 3). Individual parietal neurons (Fig. $3 A, C, E$ ) and prefrontal neurons (Fig. $3 B, D, F$ ) exhibited firing rates that varied significantly as a function of sample position (Fig. $3 A, B$ ), the categorization rule (Fig. $3 C, D$ ), and the spatial category of the sample (Fig. $3 E, F$ ).

Neurons with activity relating exclusively to the main effect of either position, rule, or category in the ANOVA/ ANCOVA were encountered in parietal cortex (Fig. $4 A, B$, black) and prefrontal cortex (Fig. $4 A, B$, gray), both in monkey 1 (Fig. $4 A$ ) and monkey 2 (Fig. $4 B$ ). There was a tendency for pure rule neurons to be more common in parietal cortex, and pure category neurons to be more common in prefrontal cortex in the two monkeys (Fig. 4A,B), and the distribution of neuronal types varied significantly between parietal and prefrontal cortex in monkey 1 (Fig. $4 A$; $\chi^{2}=8.28 ; p<0.05$ ) and in monkey 2 (Fig. $4 B ; \chi^{2}=14.39 ; p<$ 
Parietal neuron
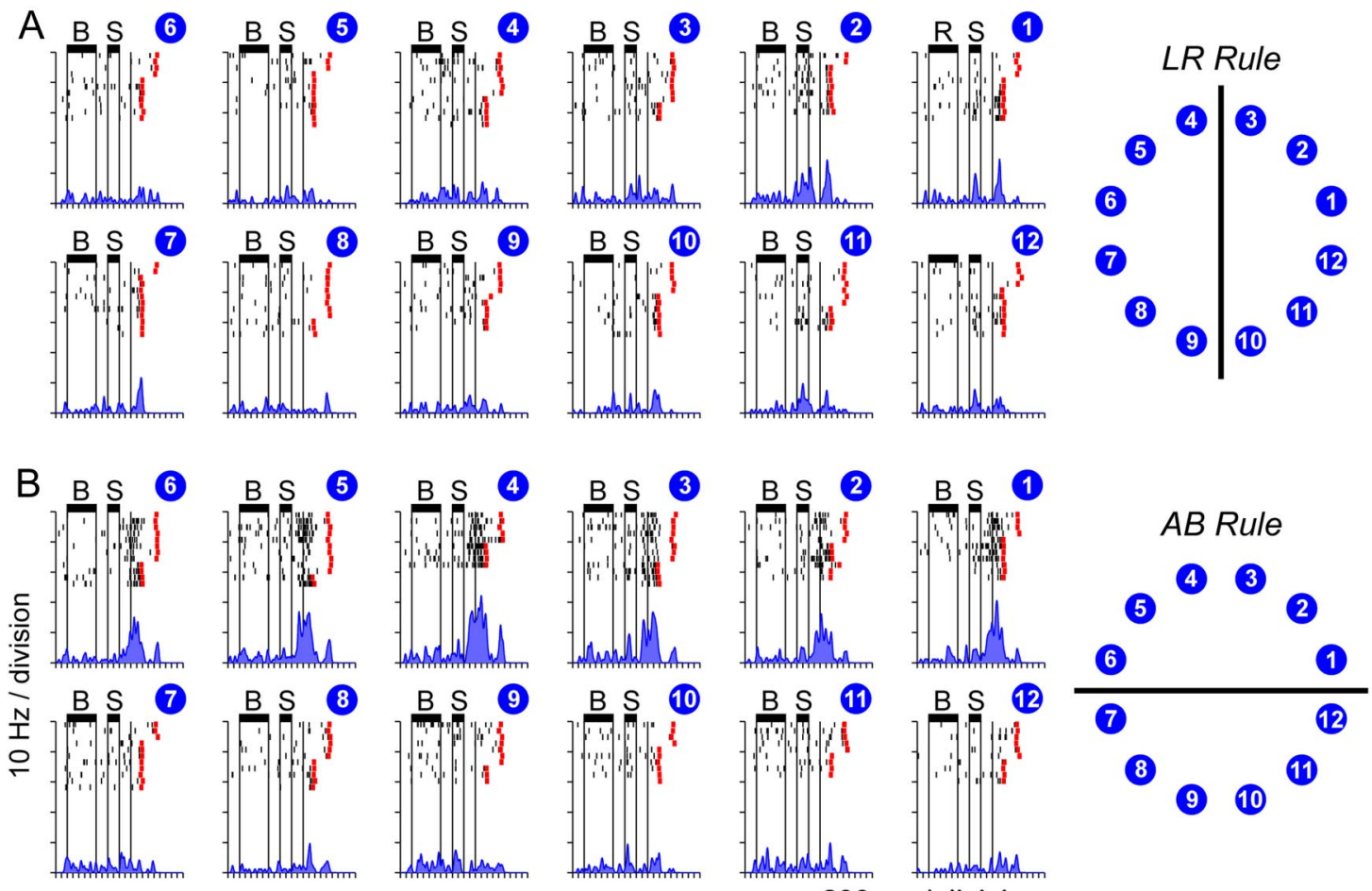

Prefrontal neuron
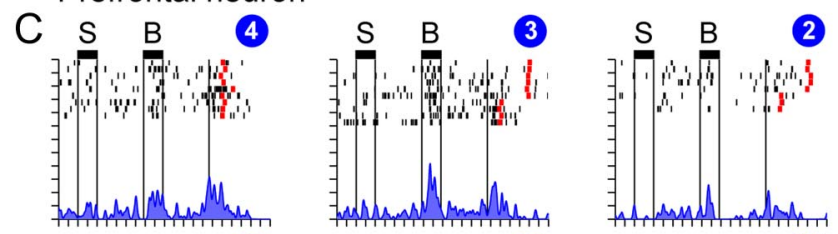

$200 \mathrm{~ms} /$ division
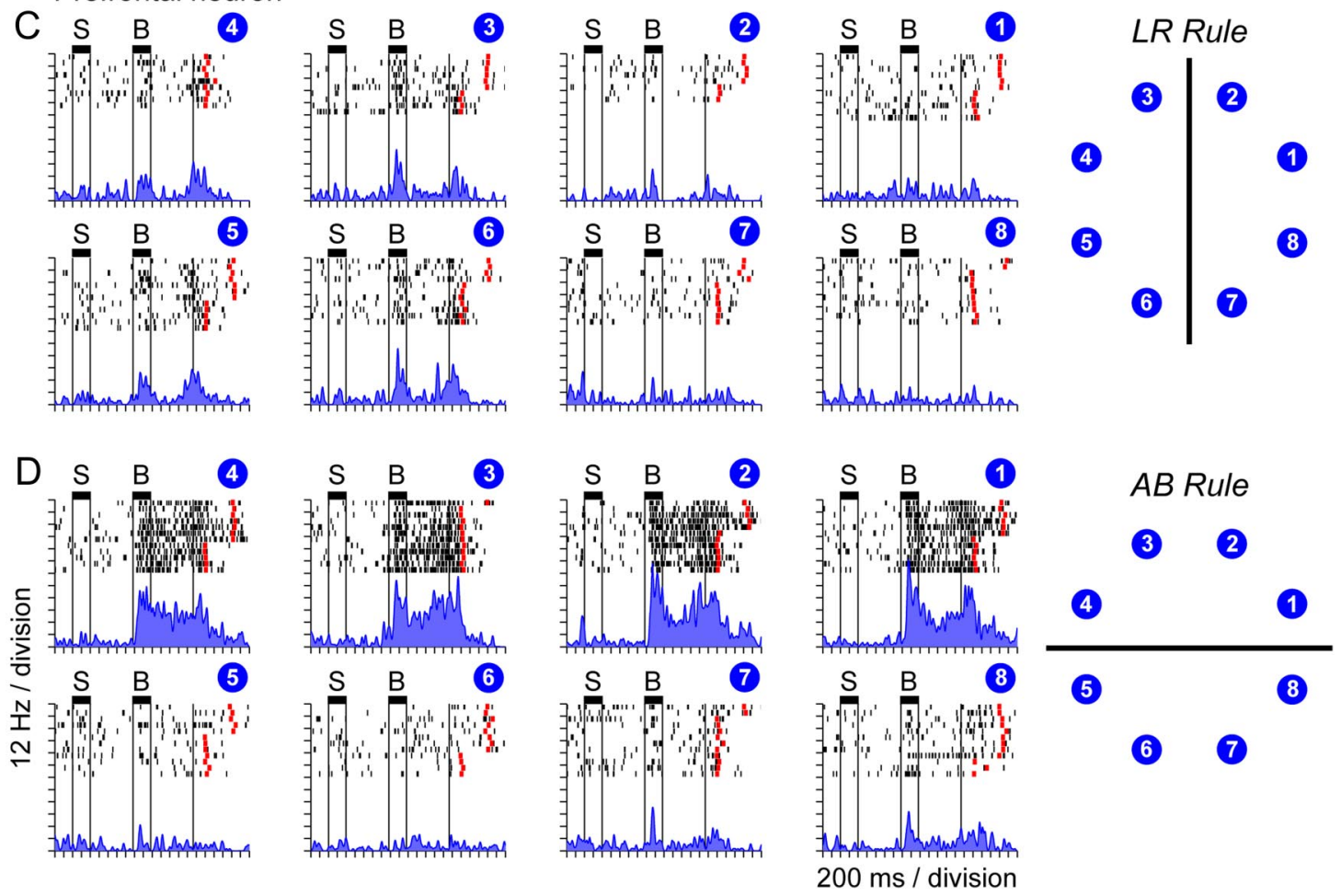

Figure 6. Rasters and spike-density functions $(\sigma=20 \mathrm{~ms})$ illustrate rule-dependent, category-selective activity of a neuron in parietal cortex $(\boldsymbol{A}, \boldsymbol{B})$ and a neuron in prefrontal cortex $(\boldsymbol{C}, \boldsymbol{D})$. The activity of both neurons was significantly influenced by the interaction between rule and category $(p<0.05)$. $A$, Activity of the parietal neuron on $L R$ rule trials (vertical boundary cue) using the boundary-sample sequence. $\boldsymbol{B}$, Activity of the same parietal neuron on $A B$ rule trials (horizontal boundary cue). The neuron exhibits a moderate increase in firing rate late in the delay period following the presentation of the sample stimulus when the sample was located in the above spatial category (positions 1-6: diagram of sample positions at right). $\boldsymbol{C}$, Activity of the prefrontal neuron on LR rule trials (vertical boundary cue) using the boundary-sample sequence. $D$, Activity of the same prefrontal neuron on AB rule trials (horizontal boundary cue). The neuron was strongly activated during the boundary period and the subsequent delay period on trials in which the sample stimulus was located in the above spatial category (positions 1-4; diagram of sample positions at right). The black bars labeled " $\boldsymbol{S}$ " and " $\boldsymbol{B}$ " indicate the sample and boundary periods, respectively. The red tick mark on each row of the rasters indicates the time at which the monkey depressed the response key. 
Parietal

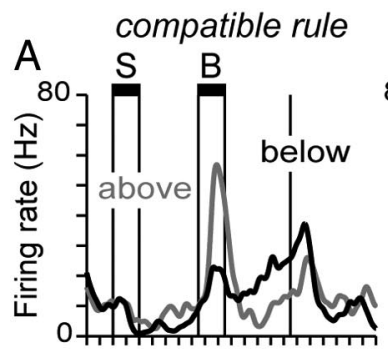

B

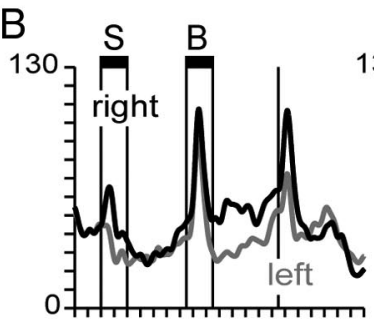

C
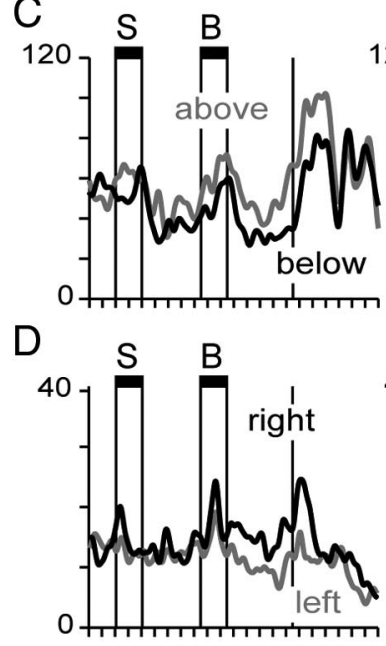

incompatible rule
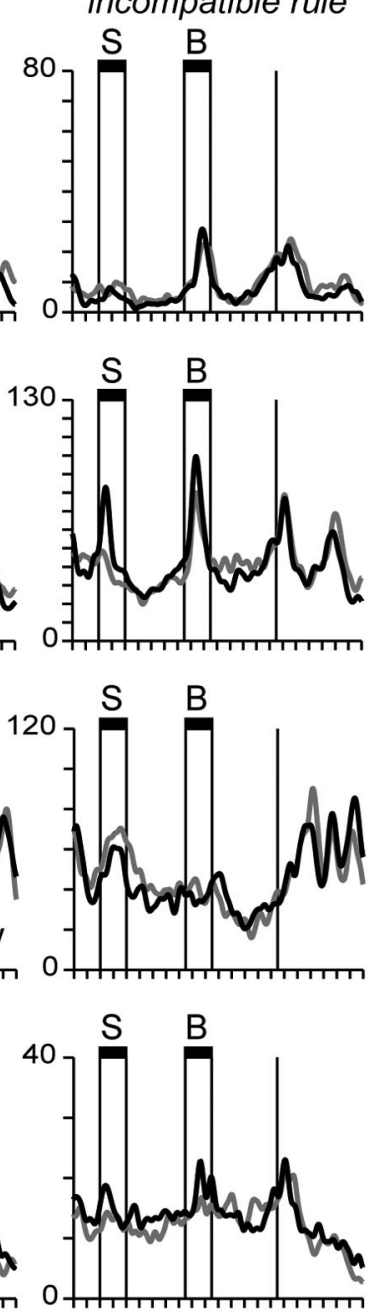

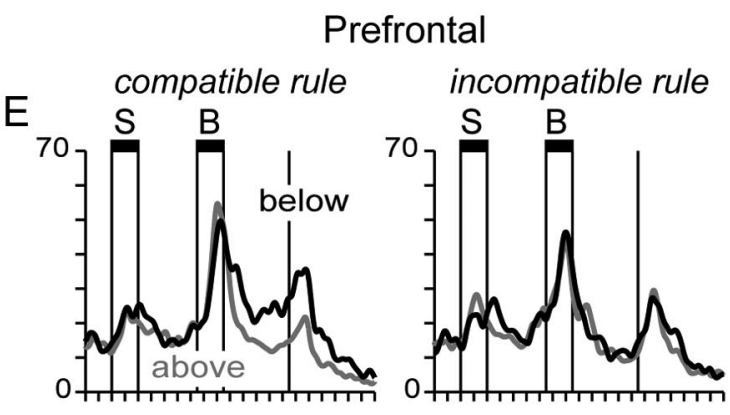

$\mathrm{F}$
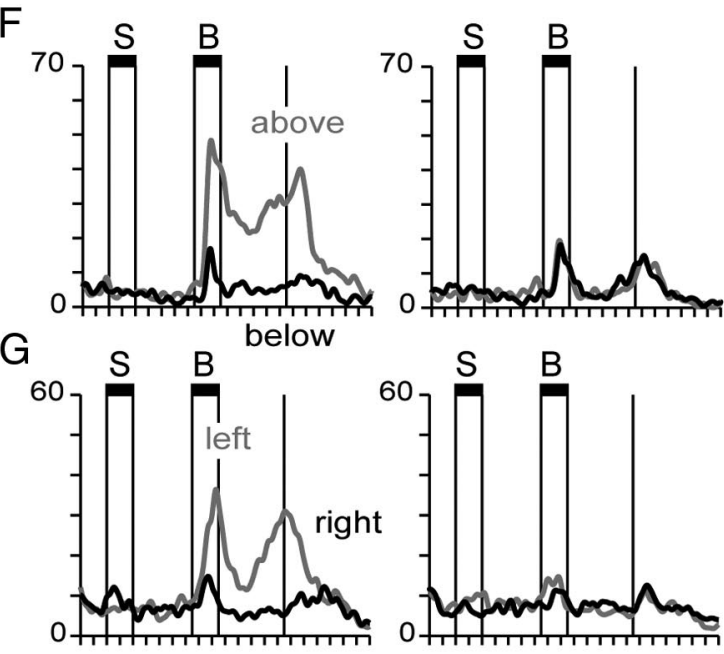

$\mathrm{H}$
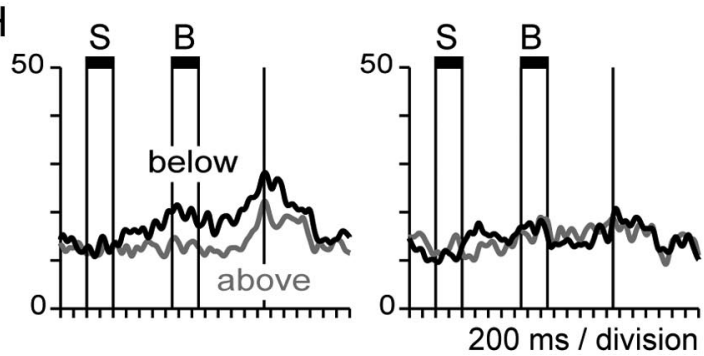

Figure 7. Spike density functions $(\sigma=40 \mathrm{~ms}$ ) illustrate the activity time course of individual neurons (4 each in parietal and prefrontal cortex) exhibiting activity that was significantly influenced $(p<0.05$ ) by the interaction between rule and either horizontal or vertical category in the ANCOVA. $\boldsymbol{A}-\boldsymbol{D}$, Plots illustrate the activity of four parietal neurons exhibiting rule-dependent category selectivity. Activity on trials when the sample was located in the right (black) and left (gray) spatial categories is plotted separately for neurons with horizontal category preferences $(\boldsymbol{B}, \boldsymbol{D})$. Activity on trials when the sample was located in the above (gray) and below (black) spatial categories is plotted separately for neurons with vertical category preferences $(\boldsymbol{A}, \boldsymbol{C}$ ). In each pair of plots in each panel, activity on compatible rule trials (e.g., the $L$ R rule for neurons preferring horizontal categories, or the AB rule for neurons preferring vertical categories) is plotted on the left, and activity on incompatible rule trials on the right. $\boldsymbol{E}-\boldsymbol{H}$, Corresponding data for four prefrontal neurons.

0.001). Considering counts of neurons including those with combinations of main effects, we obtained a similar result with an overrepresentation of rule neurons in parietal cortex and category neurons in prefrontal cortex. In this analysis, there was a significant relationship between cortical area and neuronal type in monkey $2\left(\chi^{2}=12.05 ; p<0.005\right)$, and a trend in this direction in monkey $1\left(\chi^{2}=5.10 ; p=0.07\right)$.

To compare the strength and timing of physiological signals in parietal and prefrontal cortex coding sample position, rule, and spatial category, we applied a pattern classification analysis to decode these task variables from population activity patterns measured in successive $50 \mathrm{~ms}$ time bins on trials using the sample-boundary sequence. Population signals coding the position of the sample stimulus were either earlier (Fig. 5A) and/or stronger (Fig. 5B) in parietal cortex relative to prefrontal cortex in the two monkeys (Fig. 5; dots and thicker lines indicate a significant difference in the proportion of correctly decoded trials between cortical areas). Population sig- nals coding the orientation of the category boundary (the rule) were likewise earlier and stronger within parietal cortex relative to prefrontal cortex in both monkeys (Fig. $5 C, D$ ). We obtained the reverse result in the relative strengths with which parietal and prefrontal cortex represented spatial category. Population signals reflecting the spatial category of the sample were stronger and earlier in prefrontal cortex relative to parietal cortex in both monkeys (Fig. $5 E, F$ ). These data are consistent with signals reflecting visual features of stimuli flowing in a forward direction from parietal to prefrontal cortex, and signals reflecting abstract spatial categories in our task flowing in the reverse direction, from prefrontal to parietal cortex.

Modulation of category representations by rules in the prefrontal-parietal network

Modulation of neural signals coding spatial category as a function of the rule provided a neural correlate of executive control over a spatial cognitive process. Figure 6 illustrates the rule-dependent 


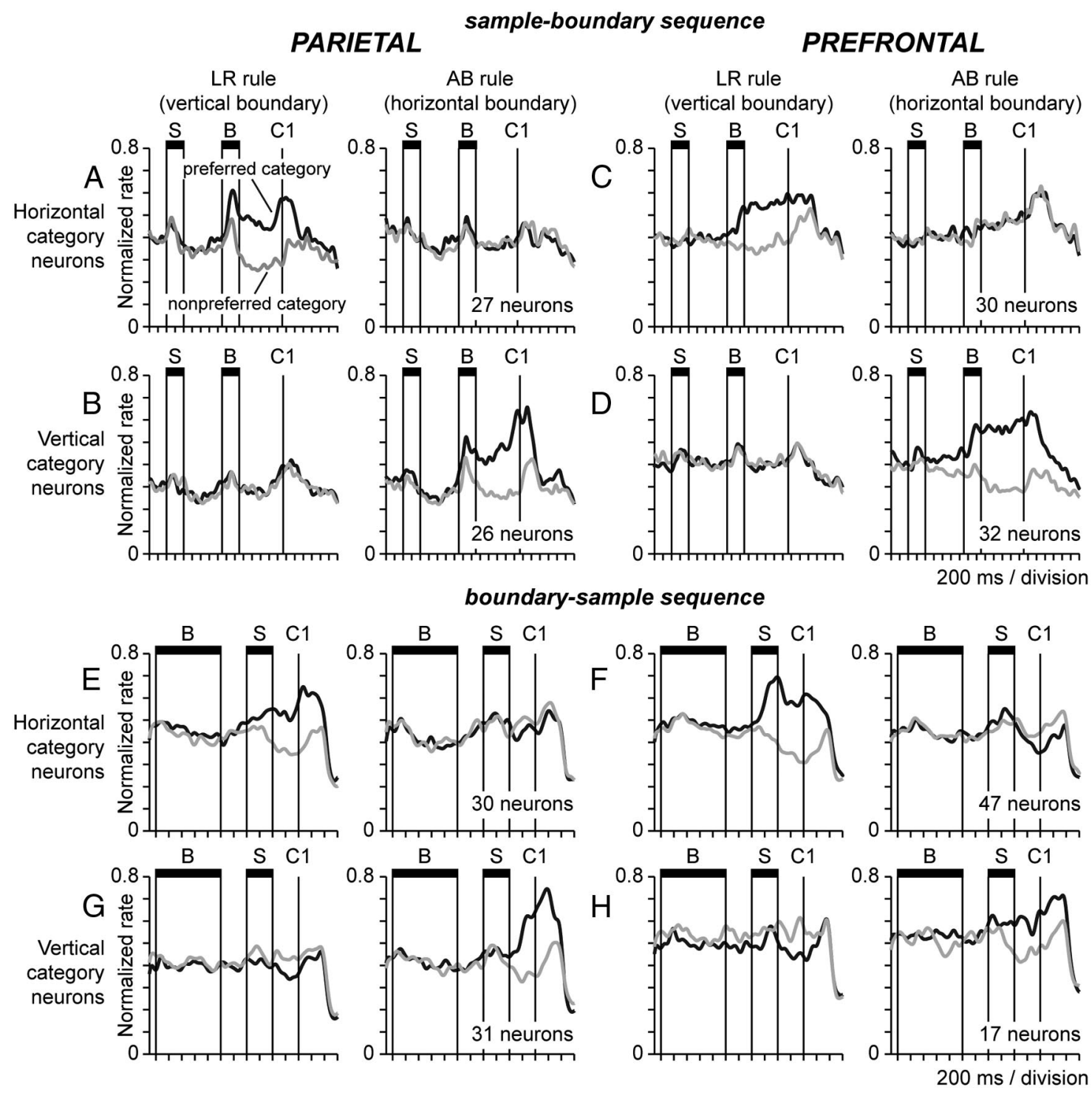

Figure 8. Average normalized population SDFs $(\sigma=40 \mathrm{~ms})$ illustrate the activity of neurons coding rule-dependent category in parietal cortex and prefrontal cortex. Separate SDFs in each plot illustrate the activity of the same neural population on trials that the sample was assigned to the preferred (black) and nonpreferred (gray) category of each neuron. Pairs of plots comprising each panel $(\boldsymbol{A}-\boldsymbol{H})$ illustrate population activity under the $L R$ and $A B$ categorization rules, respectively (the number of neurons in each population is indicated in the bottom right corner of the right plot of each pair). Neurons were included if their activity during the boundary and following delay periods related significantly $(p<0.05)$ to horizontal category and its interaction with rule, or vertical category and its interaction with rule. Neurons were excluded if their activity related to sample position $(p<0.1)$ during the sample or subsequent delay periods. The vertical lines delineate the sample period (black bar labeled " $S$ ") and the boundary cue period (black bar labeled " $B$ "), and indicate the presentation of the first choice ("C1"). $A, C$, Population SDFs illustrating the activity of neurons selective for horizontal categories under the LR rule (left) and AB rule (right) recorded using the sample-boundary sequence. $B, D$, Population activity of neurons selective for vertical categories under the LR rule (left) and AB rule (right) recorded using the sample-boundary sequence. $\boldsymbol{E}-\boldsymbol{H}$, Corresponding data for activity recorded using the boundary-sample sequence.

and category-selective activity of a neuron in parietal cortex (Fig. $6 A, B)$ and a neuron in prefrontal cortex (Fig. 6C,D). Both neurons were preferentially activated when sample stimuli appeared in any one of the positions located in the above spatial category (in the upper half of the circular stimulus array), but only when the $\mathrm{AB}$ rule was in force (Fig. $6 B, D$; numbers next to each raster correspond to stimulus positions at right). When sample stimuli were presented in the same positions under the LR rule, the preference for sample positions in the above category was no longer evident in either neuron (Fig. 6A,C). This is evidence that category-selective activity in these cells was jointly determined by stimulus position and the categorization rule. For example, category-selective activity in these neurons did not depend solely on the position of the sample stimulus, or the neurons would have been active when samples appeared in the upper positions of the stimulus array under the $\mathrm{LR}$ rule (Fig. $6 A, C$ ) as well as the $\mathrm{AB}$ rule (Fig. $6 B, D$ ). By the same token, the activity of these neurons did not depend solely on the orientation of the boundary cue either, or the neurons would have been active on all trials in which the boundary cue was horizontal, instructing the $\mathrm{AB}$ rule, and not just the subset of these trials associated with the upper category (Fig. $6 B, D$ ). This joint dependence is further indicated by the observation that the prefrontal neuron, tested with the sample-boundary sequence, did not respond immediately when the sample stimulus appeared (Fig. $6 D$, black bars labeled "S"), but rather responded later in the trial when the boundary cue had been presented (Fig. $6 D$, black bars labeled "B"), and the two pieces of information (sample position and boundary orientation) had been provided that were required to compute ruledependent category. Comparing the activity of the two neurons, 


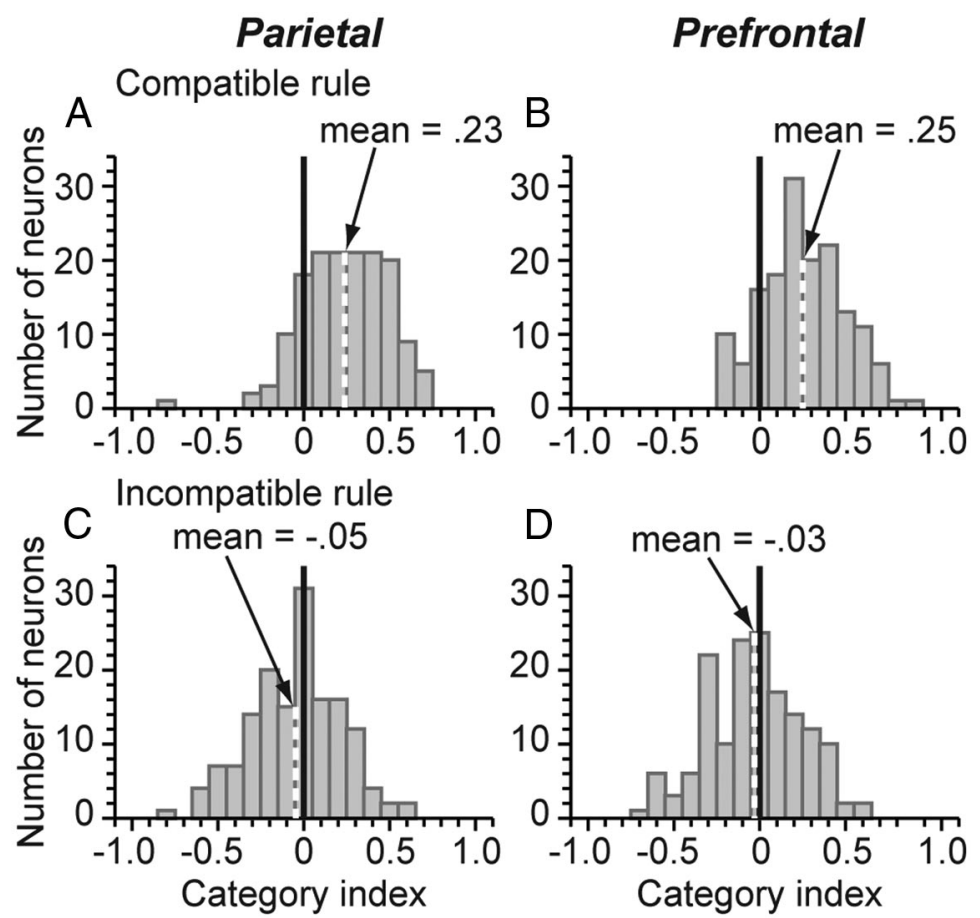

Figure 9. Distribution of the category selectivity index for single neurons recorded using the sample-boundary sequence in parietal and prefrontal cortex. Neurons were included if their activity related significantly to either horizontal or vertical category $(p<0.05$ by ANCOVA/ANOVA). The vertical white dashed line indicates the mean value of the category index in each distribution. $\boldsymbol{A}$, Distribution of category index values in parietal cortex on trials in which the rule was compatible with the category preference of each neuron. $\boldsymbol{B}$, Distribution of category index values in prefrontal cortex on compatible rule trials. $\boldsymbol{C}$, Distribution of category index values in parietal cortex on incompatible rule trials (same neural sample as in $\boldsymbol{A}$ ). $\boldsymbol{D}$, Distribution of category index values in prefrontal cortex on incompatible rule trials (same neural sample as in $\boldsymbol{B}$ ).

the prefrontal neuron exhibited notably stronger categoryselective activity (Fig. 6D) relative to the parietal neuron (Fig. $6 B)$, a pattern that is consistent with the differential representation of rule-dependent categories at the population level between the two cortical areas that we document below.

Figure 7 provides additional single-neuron examples of ruledependent and category-selective activity that were recorded in parietal (Fig. 7A-D) and prefrontal (Fig. $7 E-H)$ cortex using the sample-boundary sequence. Neurons selectively encoding horizontal categories (Fig. $7 B, D, G$ ) and vertical categories (Fig. $7 A, C, E, F, H)$ as a joint function of sample position and boundary orientation were distributed to both cortical areas.

To compare this neural signal between cortical areas at the population level, we constructed average normalized population spike density functions (SDFs) including all neurons in parietal and prefrontal cortex in which activity related significantly $(p<$ 0.05 ) to the interaction between rule and category (Fig. 8). The population activity functions parallel the single-neuron examples (Figs. 6, 7). Activity in a group of neurons in parietal and prefrontal cortex differentiated left and right spatial categories under the $\mathrm{LR}$ rule, but not under the $\mathrm{AB}$ rule (Fig. $8 A, C, E, F$ ). Activity in another, largely distinct group of neurons in both cortical areas differentiated above and below spatial categories under the $\mathrm{AB}$ rule, but not under the LR rule (Fig. $8 B, D, G, H$ ). This basic pattern was evident whether using the sample-boundary sequence (Fig. $8 A-D$ ) or the boundary-sample sequence (Fig. $8 E-H$ ). In both cases, rule-dependent category signals emerged after the second stimulus in the sequence had been presented.

Neurons with activity significantly influenced by the interaction between rule and category were encountered with compara- ble frequency in parietal and prefrontal cortex. The firing rate of $26 \%$ of parietal neurons (130 of 504) and 29\% of prefrontal neurons (142 of 496) was significantly influenced by the interaction between rule and category in monkey 1 . In monkey 2 , the firing rate of $17 \%$ of parietal neurons (86 of 512) and 16\% of prefrontal neurons (78 of 481) was significantly influenced by the interaction between rule and category.

We computed a category-selectivity index based on the one developed in prior studies of the neural correlates of categorization (Freedman et al., 2001, 2002; Roy et al., 2010). The index ranges between +1 , to indicate differences in firing rate between but not within categories, and -1 , to indicate the converse. We computed the index for each category-selective neuron on compatible and incompatible rule trials. The category index was significantly larger on compatible rule trials when the category information each neuron carried was relevant to the choice that monkeys had to make (Fig. 9A,B), than when that category information was irrelevant (Fig. 9C,D), both in parietal cortex ( $t$ test; 0.23 vs $-0.05 ; p<$ $0.0001)$, and prefrontal cortex ( $t$ test; 0.25 vs $-0.03 ; p<0.0001)$. The degree of category selectivity in prefrontal cortex measured by the index on compatible rule trials (mean index, 0.25) was comparable with that reported in prior studies of categorization (mean index, 0.16) (Roy et al., 2010).

We applied pattern classification to population activity in parietal and prefrontal cortex to generate a decoding time course that measured fluctuation in the strength of signals coding ruledependent spatial categories in the two cortical areas. To quantify the degree to which category signals exhibited rule dependence, we contrasted the accuracy of decoding on trials when the rule made the category preference of each neuron relevant or irrelevant to the required behavioral choice. For example, the activity of neurons preferring the "left" or "right" spatial categories carried task relevant category information under the LR rule, but the category information carried by these cells was irrelevant under the $\mathrm{AB}$ rule, because horizontal categories were irrelevant to choice selection under this rule. The strength of the category signal as measured at the population level by decoding accuracy was strongly modulated by the categorization rule and therefore task relevance in both cortical areas and monkeys (Fig. 10). When the category information carried by neurons was task relevant under the current rule, decoding accuracy in parietal cortex (Fig. 10, red) and prefrontal cortex (Fig. 10, blue) increased shortly after the onset of the second stimulus in the trial sequence, whether the boundary cue (Fig. $10 A, B, D, E$ ) or the sample (Fig. $10 G, H)$. Decoding accuracy peaked in the range of $80-90 \%$ correct in monkey 1 (Fig. $10 A, B ; G, H$ ) and $65-80 \%$ correct in monkey 2 (Fig. $10 D, E$ ). When the category information carried by neurons was irrelevant to the task under the current categorization rule, decoding accuracy based on the activity of the same populations of neurons was significantly reduced in parietal cortex (Fig. 10A,D,G) and prefrontal cortex (Fig. 10 B,E,H; "incom- 


\section{Decoding spatial category under compatible and incompatible rules}

MONKEY 1 PARIETAL A sample-boundary sequence

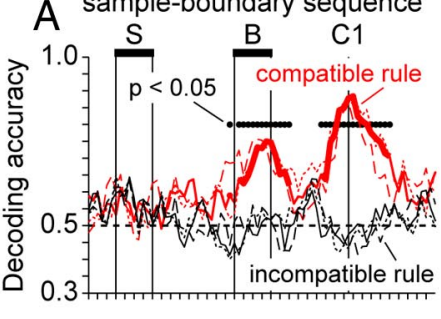

MONKEY 2

sample-boundary sequence

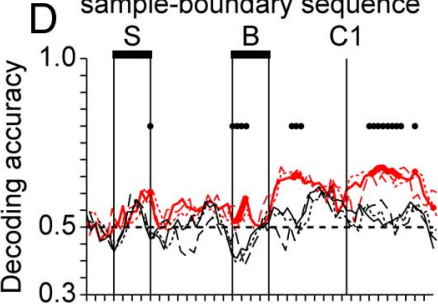

MONKEY 1

boundary-sample sequence
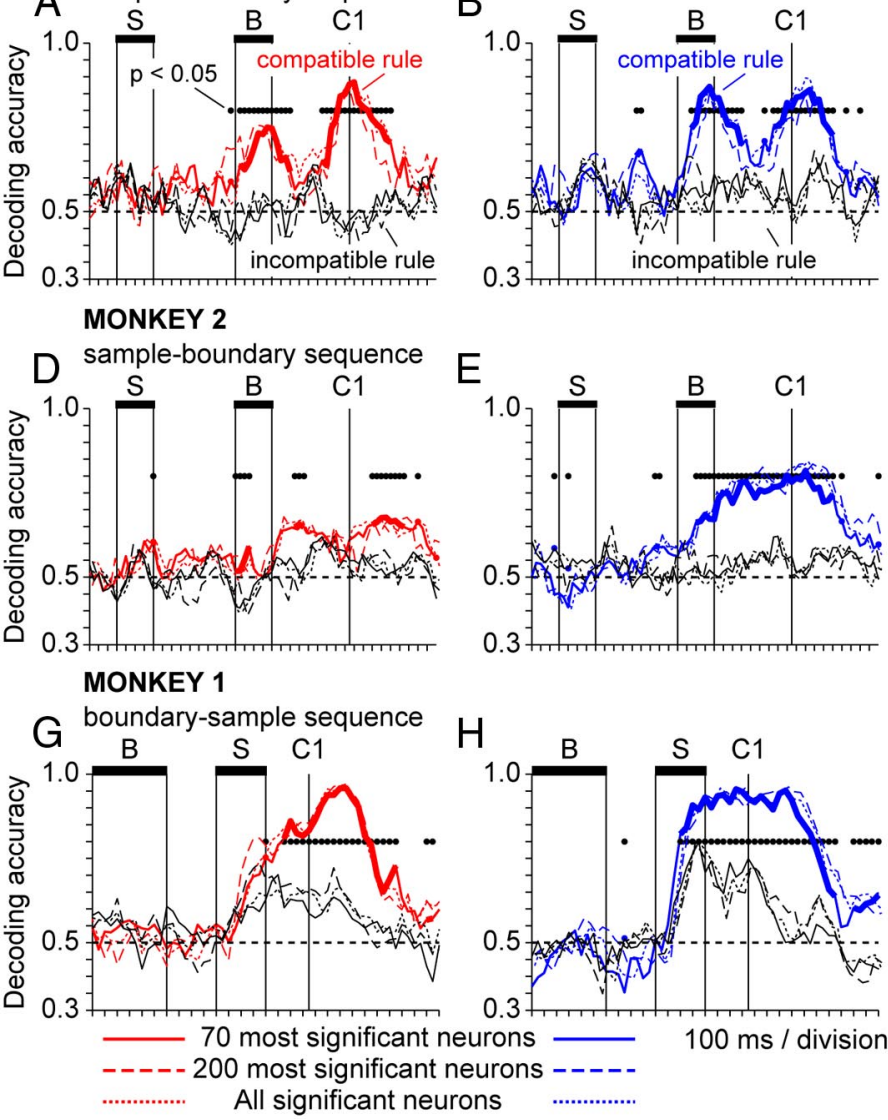

E

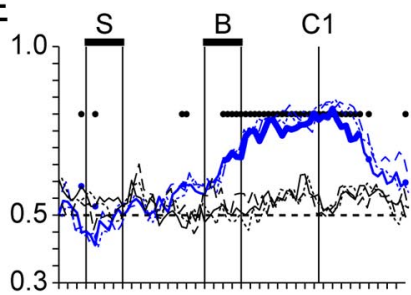

Cumulative difference in

C posterior probability (PFC - PAR)
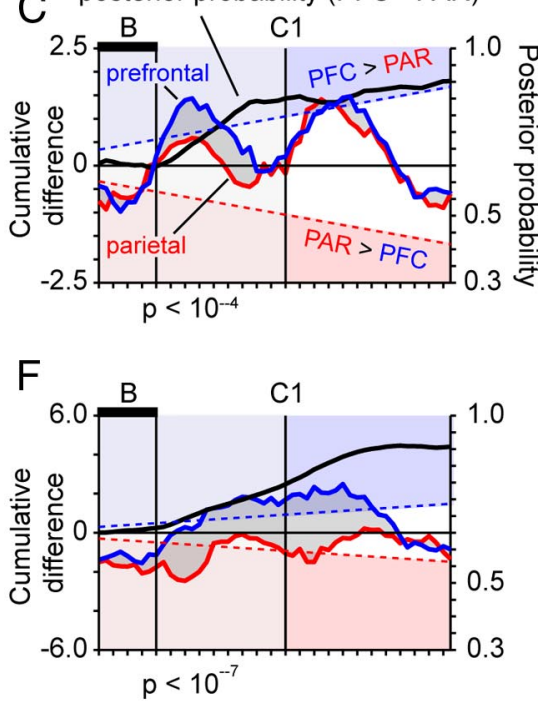

I

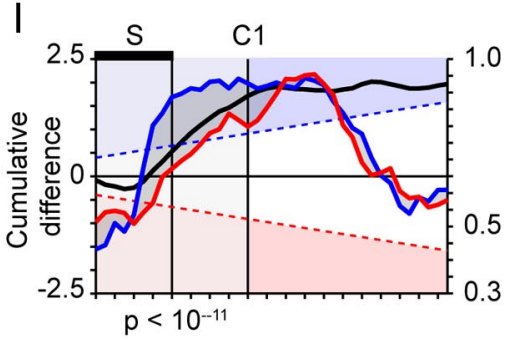

Figure 10. Decoding time courses illustrate fluctuation in the accuracy of decoding spatial category based on population activity patterns in parietal cortex (red) and prefrontal cortex (blue), measured in successive $50 \mathrm{~ms}$ time bins. Separate decoding time courses in each panel plot the results obtained using population activity on trials in which the rule was compatible with the category preference of each neuron (red and blue), and trials in which the rule was incompatible (black). Time bins in which the proportion of correctly decoded trials differed significantly between compatible and incompatible rule trials are indicated by black circles and corresponding thicker sections of the decoding time courses. Neurons in this analysis were ranked according to the $p$ value associated with the interaction between rule and category in the ANOVA/ANCOVA, and then varying numbers of the most significant neurons were selected to include in the populations used for the decoding. Decoding results indicate the accuracy obtained when based on the activity of the most significant 70 neurons (solid lines), the most significant 200 neurons (dashed lines), or all significant neurons (dotted lines) in each cortical area and monkey. $\boldsymbol{A}, \boldsymbol{B}$, Decoding accuracy obtained when using population activity in parietal cortex $(\boldsymbol{A})$ and prefrontal cortex $(\boldsymbol{B})$ of monkey 1 on sample-boundary trials. The difference between decoding accuracy on compatible and incompatible rule trials measures the modulation of category signals by the rule. For the decoding analysis including all significant neurons, 55 neurons in parietal cortex and 58 neurons in prefrontal cortex contributed. C, The blue and red time courses indicate the mean posterior probability over time (averaged over trials) associated with the correct spatial category on compatible rule trials, based on neural activity in prefrontal and parietal cortex, respectively (data from monkey 1 on sample-boundary trials). The black time course illustrates the cumulative difference between the two time series. The diagonal dashed lines indicate the upper and lower confidence boundaries established by the sequential trials test. The cumulative difference function crosses the upper confidence boundary, indicating that the posterior probability is significantly larger in prefrontal cortex relative to parietal cortex $(p<0.05)$. $\mathbf{D}-\boldsymbol{F}$, Corresponding data from monkey 2 on sample-boundary trials. For the decoding analysis including all significant neurons, 62 neurons in parietal cortex and 90 neurons in prefrontal cortex contributed. G-I, Corresponding data from monkey 1 on boundary-sample trials. For the decoding analysis including all significant neurons, 104 neurons in parietal cortex and 120 neurons in prefrontal cortex contributed.

patible rule," black time courses). Time bins in which the proportion of correctly decoded trials differed significantly $(p<$ 0.05 ) as a function of the categorization rule are indicated by black circles and thicker sections of the decoding time courses (Fig. 10; the large majority of time bins with significant rule modulation cluster in the period following the presentation of the second stimulus in the trial sequence). We obtained comparable decoding results whether decoding was based on the firing rates of the top 70 (Fig. 10; solid lines), the top 200 (Fig. 10; dashed lines), or all significant neurons (Fig. 10; dotted lines) in each cortical area, providing evidence that the decoding results were representative of the signals present within parietal and prefrontal cortex. Rule modulation of category signals did not seem to be contingent on the visual properties of the stimuli that elicited these effects, namely the sample or boundary stimuli, as rule modulation could occur following the presentation of either stimulus. In the sample-boundary data, decoding accuracy was generally near chance levels $(50 \%$ correct) on trials when the rule made the category preference of neurons irrelevant to the required choice (Fig. 10A, $B, D, E$; incompatible rule). On boundary-sample trials, decoding accuracy remained above chance levels on incompatible rule trials (Fig. 10G,H). The more accurate decoding on incompatible rule trials under the boundary-sample sequence suggests that signals reflecting the category of visible stimuli (Fig. $10 G, H$ ) are less completely modulated by rules than signals reflecting the category of stimuli stored in working memory (Fig. $10 A, B, D, E$ ). 
MONKEY 1 Category

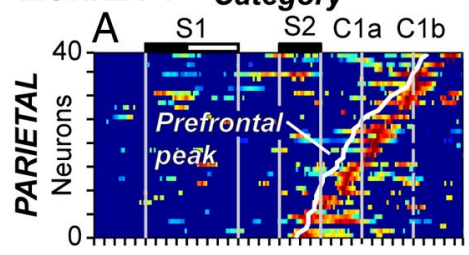

C

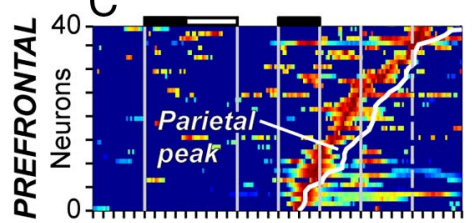

MONKEY 2 Category

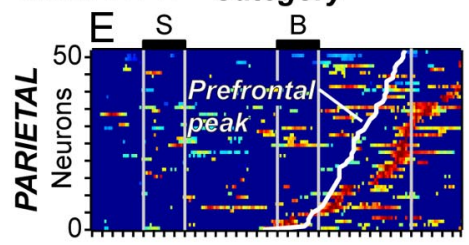

$\mathrm{G}$

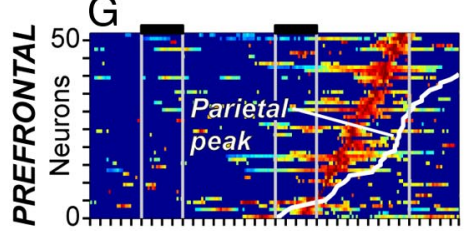

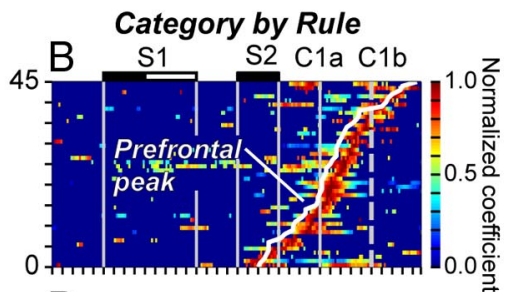

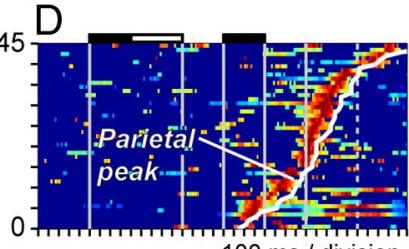

Category by Rule

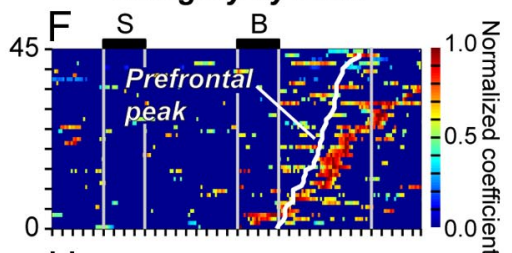

$\mathrm{H}$

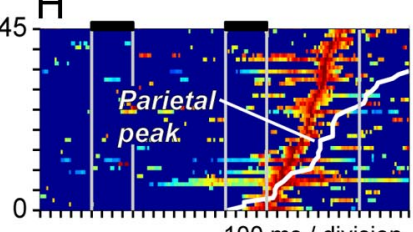

$100 \mathrm{~ms} /$ division

Figure 11. Time course of regression coefficients obtained for category and the interaction between category and rule for individual neurons in parietal and prefrontal cortex. Each row in the color plots indicates the time series of regression coefficients obtained for a single neuron normalized to the peak coefficient for that neuron. In monkey 1, data from sample-boundary and boundary-sample trials are aligned to the onset of the second stimulus ("S2") and combined. (The two data sets shared the same duration of S2. The different durations of the first stimulus, S1, included in these data are indicated by the black and white portions of the bar marked "S1." The different timings of the onset of the first choice are indicated by vertical lines marked "C( $\boldsymbol{a}^{\text {" and }}$ "C1b.") $\boldsymbol{A}, \boldsymbol{B}$, Regression coefficients for category $(\boldsymbol{A})$ and the interaction between rule and category $(\boldsymbol{B})$ obtained for parietal neurons in monkey 1. The white lines show the time of peak coefficients in prefrontal cortex (Prefrontal peak) of the same monkey for comparison. $\boldsymbol{C}, \boldsymbol{D}$, Regression coefficients for category $(\boldsymbol{C})$ and the interaction between rule and category $(\boldsymbol{D})$ obtained for prefrontal neurons in monkey 1. The white lines show the time of peak coefficients in parietal cortex (Parietal peak) of this monkey for comparison. The dark blue regions indicate time points at which coefficients were nonsignificant. $\boldsymbol{E}-\boldsymbol{H}$, Corresponding data for monkey 2.

In addition to classifying trials to categories based on patterns of population activity observed in each time bin, the decoding analysis also provided the posterior probability, or confidence, associated with each classification. We constructed time courses of the mean posterior probability in the decoding analysis associated with the correct category on compatible rule trials using the activity of the same populations of neurons (Fig. 10C, F, I). The mean posterior probability was greater and/or increased earlier in prefrontal cortex (Fig. 10C, F, I; blue time course) relative to parietal cortex (Fig. 10C, F, I; red time course), indicating a stronger representation of the correct rule-dependent category in prefrontal cortex. We applied the sequential trials test (Armitage, 1975) to determine whether differences in the posterior probability time series differed significantly between parietal and prefrontal cortex. The test evaluates significance by determining whether the cumulative difference between two time series crosses one of two $95 \%$ confidence boundaries centered around a mean difference of 0 (the distance between boundaries increases over time to compensate for the increasing probability of accumulating larger differences between the series over time by chance). Applied to the posterior probability time series, the test

confirmed that posterior probabilities associated with the correct rule-dependent category in each trial were significantly greater in prefrontal cortex relative to parietal cortex in each monkey, and using both stimulus sequences (Fig. 10C, F, I; the cumulative difference functions, shown as thick black lines, increase monotonically upward to cross the upper boundary indicating significantly larger values in prefrontal cortex). To confirm this result, we computed posterior probability based on firing rates in a $150 \mathrm{~ms}$ time bin advanced in $150 \mathrm{~ms}$ steps (so that the same action potentials did not contribute to successive values), and then tested the significance of the difference between parietal and prefrontal cortex in the mean posterior probability observed during the second stimulus and subsequent delay periods (by $t$ test). The mean posterior probability associated with the correct ruledependent spatial category was significantly greater in prefrontal cortex than parietal cortex in monkey 1 on sample-boundary trials $\left(0.62\right.$ vs $\left.0.57 ; p<10^{-4}\right)$, monkey 2 on sample-boundary trials ( 0.59 vs $0.55 ; p<$ $10^{-7}$ ), and monkey 1 on boundary-sample trials $\left(0.74\right.$ vs $\left.0.63 ; p<10^{-11}\right)$.

To evaluate whether the depth of modulation of category signals as a function of rule varied significantly across cortical areas, we performed a two-way ANOVA on the posterior probability associated with the spatial category of the sample using cortical area and rule compatibility as factors. (The rule compatibility factor contrasted posterior probabilities computed using activity on separate subsets of trials in which the rule was compatible or incompatible with the category preference of each neuron.) We combined the boundary-sample and sample-boundary data in monkey 1 for this analysis and performed the decoding in both monkeys based on firing rates measured within a $150 \mathrm{~ms}$ time bin advanced in $150 \mathrm{~ms}$ steps (during the second stimulus and subsequent delay period). We detected a significant interaction between cortical area and rule compatibility in the analysis, with stronger rule effects on category signals in prefrontal cortex, both in monkey $1(\mathrm{~F}=5.72 ; p<0.05)$, and monkey $2(\mathrm{~F}=8.02$; $p<0.005)$.

To quantify the influence of rule, category, and their interaction on the activity of single neurons, we measured the firing rate of each neuron (without preselection) within a sliding $200 \mathrm{~ms}$ window advanced in $20 \mathrm{~ms}$ steps, and regressed firing rate over trials at each time step onto category, and the interaction between category and rule. The regression analysis produced a time series of regression coefficients for each neuron showing the strength of the relationship between firing rate and spatial category, as well as the interaction between category and rule, at each time step (Fig. 11). We found by this analysis that neurons tended to exhibit their strongest relationship to category and the interaction with rule for a relatively short duration, and that the time at which this occurred was distributed in the interval after the onset of the 
second stimulus and the first choice stimulus in the trial. Ranking neurons according to the time of the peak coefficient, early to late from bottom to top in Figure 11 , produced a diagonal band of color in each plot indicating the rate at which neurons were recruited to represent category or its interaction with rule in each cortical area. Pairs of plots illustrating recruitment in parietal and prefrontal cortex for a given variable include equal numbers of neurons in the two areas so the slopes of the lines of color indicating neuronal recruitment can be visually compared across areas. Neurons with activity reflecting spatial categories (Fig. $11 A, C ; E, G$ ) and the interaction between rule and category (Fig. $11 B, D ; F, G$ ) were recruited at a faster rate or at earlier times in the trial in prefrontal cortex compared with parietal cortex, in both monkey 1 (Fig. $11 A-D$ ) and monkey 2 (Fig. $11 \mathrm{E}-\mathrm{H}$ ).

We compared the mean population regression coefficient for the interaction between rule and category obtained in parietal and prefrontal cortex at each time point in the trial (Fig. 12). (Mean population coefficients are small because all neurons in each population were included in computing the population average, and only a few neurons in the population were active at any one time, so that most of the coefficients contributing to the population average at each time point were nonsignificant and therefore 0 .) We restricted this analysis to the neurons with the strongest signals, in which the peak coefficient was significant at $p<0.01$ and was $>3 \mathrm{~Hz}$. We found that the rising edge of the increase in the mean population coefficient for the interaction between rule and category lead in prefrontal cortex and followed in parietal cortex (Fig. 12A,B), and that the regression coefficient for the interaction was significantly larger in prefrontal cortex than parietal cortex (Fig. $12 C, D$; sequential trials test). To confirm the significance of this difference between cortical areas, we reran the regression analysis using a $100 \mathrm{~ms}$ time bin advanced in 100 ms steps, and found that the mean population coefficient for the interaction between rule and category during the second stimulus and subsequent delay periods was significantly larger (by $t$ test) in prefrontal cortex than parietal cortex, both in monkey $1(0.69$ vs $0.47 ; p<0.05)$ and in monkey 2 ( 0.99 vs $\left.0.49 ; p<10^{-9}\right)$.

To evaluate the significance of differences in the timing of this signal between cortical areas, we compared the mean time to the half-maximum coefficient for the interaction between rule and category in parietal and prefrontal neurons. In monkey 1 , the time to the half-maximum coefficient in prefrontal cortex (Fig. $12 \mathrm{E}, \mathrm{G}$; blue bars and line; mean, $582.7 \mathrm{~ms}$ after onset of the second stimulus) was significantly earlier than the time to halfmaximum in parietal cortex (Fig. 12E, G; red bars and line; mean,

\section{Category by Rule Interaction} MONKEY 1
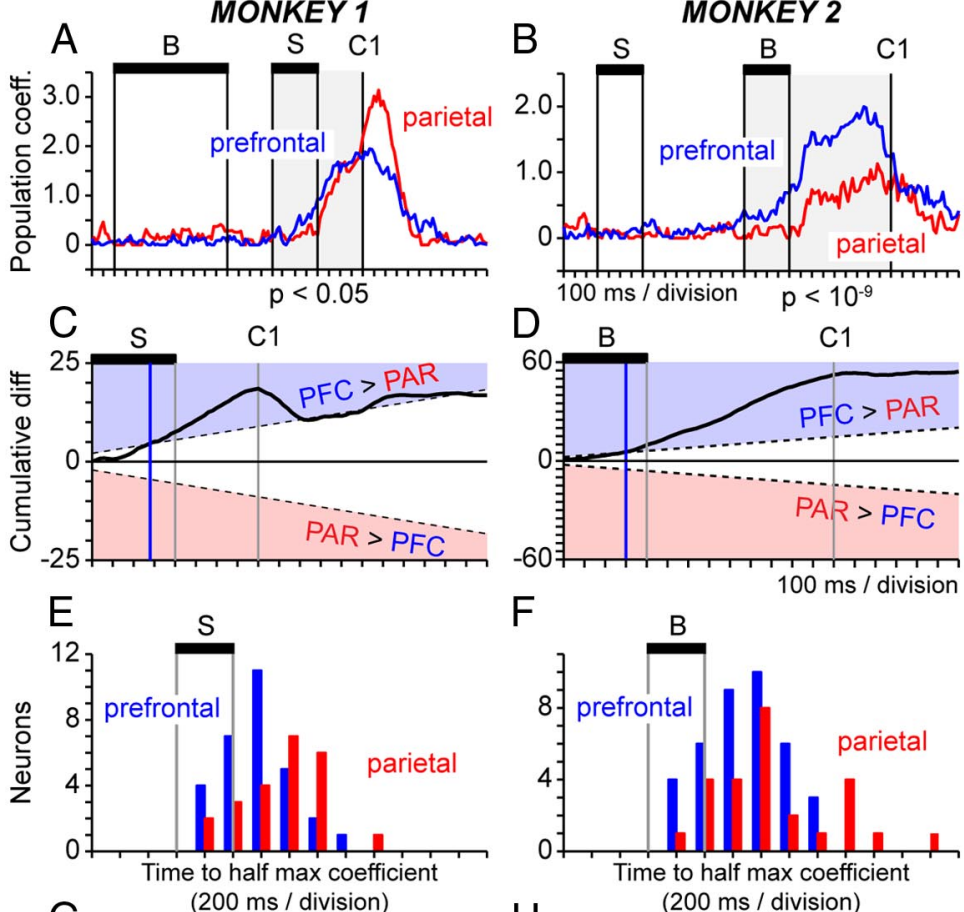

$\mathrm{F}$
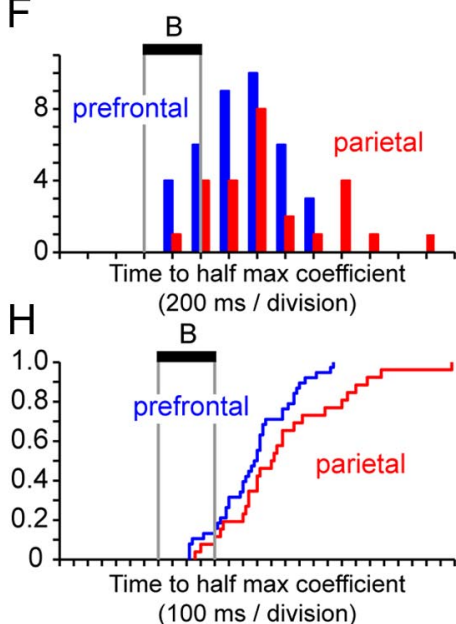

$\begin{array}{ll}(100 \mathrm{~ms} / \text { division }) & \text { (100 ms / division) }\end{array}$

Figure 12. Comparison of the strength and timing of regression coefficients obtained for the interaction between rule and category in parietal and prefrontal neurons (data restricted to regression coefficients that were significant at $p<0.01$ ). $A, B$, Plots stimulus in the trial sequence. The thick black lines indicate the cumulative difference between population coefficient time series in the two cortical areas (prefrontal - parietal). The cumulative difference functions deflect upward and cross the upper conficategory is significantly larger in prefrontal cortex ( $p<0.05$, minimum effect size of 0.8 ). $\boldsymbol{E}, \boldsymbol{F}$, Frequency distribution 列 f-maximum regression coefficient for the interaction between rule and category in prefrontal cortex (blue lines) and parietal cortex (red lines) of monkey $1(\boldsymbol{G})$ and monkey $2(\boldsymbol{H})$.

$726.1 \mathrm{~ms} ; \mathrm{t}=2.05 ; p<0.05)$. Likewise in monkey 2 , the time to the half-maximum coefficient in prefrontal cortex (Fig. $12 \mathrm{~F}, \mathrm{H}$; blue bars and line; mean, $679.5 \mathrm{~ms}$ ) was significantly earlier than the time to half-maximum coefficient in parietal cortex (Fig. $12 \mathrm{~F}, \mathrm{H}$; red bars and line; mean, $886.15 \mathrm{~ms} ; \mathrm{t}=2.33$; $p<0.05$ ).

Although the neurophysiological signal reflecting the interaction between rule and category was significantly earlier in prefrontal cortex than parietal cortex in both monkeys, neurons exhibiting this signal were coactivated to a considerable degree in the two areas, providing evidence that the executive control of categorization was a distributed process. Furthermore, it is important to note that it is not possible by single-neuron recording to determine whether the first neurons carrying the signal reflecting the interaction between rule and category were located in prefrontal or parietal cortex. Rather, these data provide evidence 


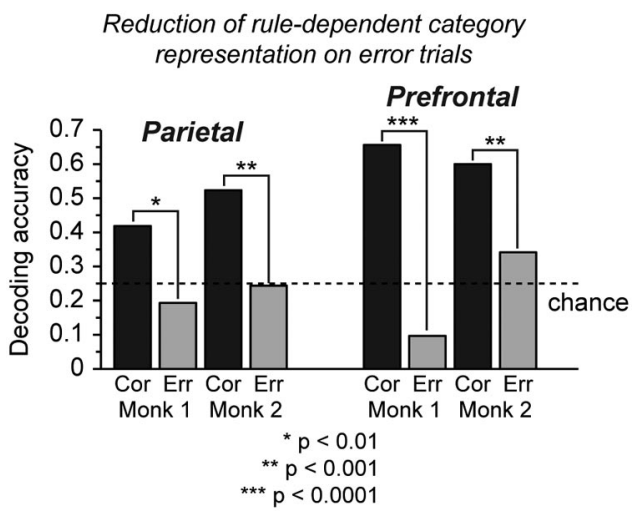

Figure 13. Relationship of population decoding accuracy to behavioral performance. In this analysis, population activity was used to decode rule-dependent spatial category defined jointly by sample position and boundary orientation and coded as a categorical variable with four levels (left, right, above, and below). Decoding is based on the 50 neurons in each cortical area and monkey having activity most significantly related to the interaction between rule and category. Decoding accuracy was significantly reduced on error trials (gray) relative to correct trials (black), both in parietal cortex and prefrontal cortex in monkeys 1 and 2 considered individually. The significance of the difference in decoding accuracy on correct and error trials, evaluated using the $z$ test of proportions, is as indicated by asterisks in the figure. Chance decoding (given 4 categories) is $25 \%$ correct.

that on average, neurons with stronger signals were more rapidly recruited to reflect the interaction between rule and category in prefrontal cortex.

\section{Relationship of rule-dependent category representation to successful performance}

To determine whether the strength of neural signals reflecting the executive control of categorization related to behavioral performance, we compared the accuracy of decoding rule dependent category as a single variable with four levels (left, right, above, below) using neural activity on correct and error trials. We found that decoding accuracy was significantly reduced on error trials (Fig. 13, gray) relative to correct trials (Fig. 13, black) in both cortical areas and monkeys. Erroneous choices were therefore preceded by a failure of the prefrontal-parietal network to accurately encode the correct rule-dependent spatial category of the sample.

\section{Discussion}

Executive control involves determining not only what action to take, but what cognitive process to execute to select the best action in a given context. Our objective in this experiment was to better understand how physiological signals in prefrontal cortical networks mediate executive control over cognitive processing. To approach that goal, we developed a task in which monkeys had to selectively implement competing cognitive processes in response to a rule cue. We then sought to isolate neural signals that reflected rule-dependent cognitive processing in the prefrontalparietal network. The task we developed for this purpose required monkeys to flexibly reassign the same circular array of stimulus positions into different spatial categories according to different categorization rules or grouping criteria on a trial-by-trial basis. This task allowed us to isolate neural signals in the network that were selective for spatial categories and were also rule dependent, providing a cellular correlate of executive control over a cognitive process. By characterizing the timing and distribution of this physiological signal in the prefrontal-parietal network, we sought to understand further how the network implemented the com- putational flexibility the task required, and what the respective roles of parietal and prefrontal cortex in this computational flexibility might be.

We report several primary findings. First, the spatial variables defined by the DYSC task - spatial position, rule (boundary orientation), and spatial category-were each represented at the cellular level by the parallel activation of neurons with similar physiological properties in parietal and prefrontal cortex. Neural representations related to task performance therefore were fundamentally distributed in this network. However, population decoding revealed that signals reflecting the spatial attributes of visual stimuli (sample position and boundary orientation) emerged earlier and/or were stronger in parietal cortex (area 7a) relative to prefrontal cortex (area 46), consistent with a feedforward of visually evoked signals in the network. Conversely, we found that signals reflecting the spatial category of the sample were stronger and emerged earlier in prefrontal cortex relative to parietal cortex, consistent with a feedback of abstract category signals in the network. This suggests that, within the context of this task, the spatial category signal may originate in prefrontal cortex.

Second, a primary objective of the current experiments was to better understand the neural mechanism by which the brain was able to assign one stimulus to multiple alternative categories according to a variable rule, and we detected a direct neural correlate of this computational flexibility. We found that single neurons exhibited activity that was both category selective and also rule dependent (Figs. 6-12). Neurons of this class were activated in the case that the sample stimulus was assigned to the preferred spatial category of each neuron, but only when that category was compatible with the rule in force, in which case the category information carried by neurons was task relevant and provided a basis to select the correct choice. This contextdependent activation of neuronal populations coding categories appears to reflect executive control over a cognitive process, as opposed to a sensorimotor process, in the sense that rulemodulated neurons encoded abstract information (a spatial category) rather than sensorimotor information (such as the position of a stimulus or direction of forthcoming movement). We found that rule-modulated category neurons were distributed between prefrontal and parietal cortex, suggesting that the executive control of spatial categorization was a network-level process, involving at a minimum these two cortical areas (and most probably others).

Third, by comparing the strength and timing of rulemodulated category signals in the prefrontal-parietal network, we obtained evidence that executive control over spatial categorization in our task may originate within the prefrontal cortex. Specifically, we found that neural signals coding rule-dependent spatial categories (1) were stronger, (2) more deeply modulated by the rule, and (3) emerged earlier in prefrontal cortex relative to parietal cortex. The conclusion that signals reflecting the executive control of categorization were stronger and earlier in prefrontal cortex was strengthened by the fact that these observations were confirmed significant in each of the two monkeys considered individually. However, it is important to note some aspects of executive control as defined by our task were represented by neural signals that emerged in parietal cortex first, notably the signal reflecting the categorization rule, although this signal may have reflected the visual attributes of the boundary cue instructing the rule, rather than an abstract rule per se.

Prior work on the neural mechanisms of categorization in the somatosensory system has shown that neural signals reflecting 
the category of vibrotactile stimuli are distributed between the supplementary motor area (SMA) and pre-SMA (Romo et al., 1993, 1997). Prior work in visual categorization has shown that neurons coding object categories based on shape are distributed between prefrontal and inferotemporal cortex (Freedman et al., 2001, 2002, 2003; Freedman and Miller, 2008), whereas neurons coding categories based on the spatial attributes of visual stimuli are distributed between prefrontal and posterior parietal cortex (Freedman and Assad, 2006; Merchant et al., 2011). Regarding spatial categorization, a recent study compared the timing and strength of category-selective neural signals in parietal and prefrontal cortex of a monkey assigning horizontal bar stimuli to the spatial categories "high" and "low" (Merchant et al., 2011). That study and the present one both found that category-selective signals were stronger and arose earlier in prefrontal cortex relative to parietal cortex. Merchant et al. (2011) found that category signals in parietal cortex emerged only toward the end of the trial near the time of the categorical choice, whereas we observed an earlier recruitment of parietal cortex and a more extensive period of prefrontal-parietal coactivation (Figs. 8, 10, 12, 13) (but see Fig. $6 \mathrm{~A}$ for a parietal neuron with a late-onset category response). A recent study in which monkeys assigned visual stimuli to spatial categories based on their direction of visual motion observed the opposite order of recruitment in the prefrontal-parietal network, with neurons in parietal area LIP exhibiting stronger and earlier category-selective signals relative to their prefrontal counterparts (Swaminathan and Freedman, 2011). The reason for the differences in network dynamics observed in these studies is not clear. One possibility could be that parietal area 7a [the focus of recording in the present study and that of Merchant et al. (2011)] and parietal area LIP [the focus of recording in the study by Swaminathan and Freedman (2011)] are recruited to represent spatial categories at different times relative to prefrontal cortex. Another possibility is that parietal cortex leads prefrontal cortex in category representation to the degree that visual features and visual categories are tightly coupled, as visually derived information generally flows from parietal to prefrontal cortex (Fig. 5A-D). In the present study, spatial position and spatial category were dissociated by requiring that monkeys use two sequentially presented stimuli to compute category, and further by assigning each sample stimulus (and hence each collection of stimulus features) to two alternative categories over trials. Thus, the requirement to compute category using information stored in working memory, or the requirement to do this flexibly in accordance to a changing rule, could shift network dynamics in favor of prefrontal cortex.

Other work on the neural mechanisms of executive control has shown that neurons participating in rule-dependent computations are found both within posterior parietal cortex (Stoet and Snyder, 2004; Asari et al., 2005; Kamigaki et al., 2009, 2011) and prefrontal cortex (Wallis et al., 2001; Nakahara et al., 2002; Mansouri et al., 2006; Muhammad et al., 2006). A recent study of particular relevance characterized the neural mechanisms of executive control in prefrontal cortex specifically as it applied to object categorization (Roy et al., 2010). Both that prior study and the present one found that, when monkeys had to flexibly assign the same visual stimulus to competing perceptual categories, the alternative categories were represented by largely distinct populations of prefrontal neurons that were conditionally activated as a function of the category boundary applied. The agreement between these two studies suggests that prefrontal cortex contains a common neural mechanism for flexible categorization that applies to both spatial and object categories.
Characterizing differences in the strength and timing of physiological signals that are distributed across interconnected cortical areas provides one approach to disentangling the contributions that each cortical area makes to their shared patterns of electrical activity (Chafee and Goldman-Rakic, 1998; Crowe et al., 2004; Nieder and Miller, 2004; Buschman and Miller, 2007). Our present data provide evidence that signals reflecting the interaction between rules and categories, and therefore the executive control of categorization as a cognitive process, are earlier and stronger in prefrontal cortex relative to posterior parietal cortex. These findings may provide a positive image of the cognitive deficit in executive control that has long been recognized as a hallmark of prefrontal cortical damage in humans (Goldman-Rakic, 1987; Miller, 2000), by providing some of the first single-neuron evidence that prefrontal cortex might be a generator of distributed signals that reflect computational flexibility in cortical networks.

\section{References}

Armitage P (1975) Sequential medical trials, Ed 2. Oxford: Blackwell.

Asari T, Konishi S, Jimura K, Miyashita Y (2005) Multiple components of lateral posterior parietal activation associated with cognitive set shifting. Neuroimage 26:694-702.

Ashby FG, Maddox WT (2005) Human category learning. Annu Rev Psychol 56:149-178.

Averbeck BB, Crowe DA, Chafee MV, Georgopoulos AP (2003) Neural activity in prefrontal cortex during copying geometrical shapes. II. Decoding shape segments from neural ensembles. Exp Brain Res 150:142-153.

Buschman TJ, Miller EK (2007) Top-down versus bottom-up control of attention in the prefrontal and posterior parietal cortices. Science 315:1860-1862.

Cavada C, Goldman-Rakic PS (1989) Posterior parietal cortex in rhesus monkey. II. Evidence for segregated corticocortical networks linking sensory and limbic areas with the frontal lobe. J Comp Neurol 287:422-445.

Chafee MV, Goldman-Rakic PS (1998) Matching patterns of activity in primate prefrontal area $8 \mathrm{a}$ and parietal area 7ip neurons during a spatial working memory task. J Neurophysiol 79:2919-2940.

Chafee MV, Goldman-Rakic PS (2000) Inactivation of parietal and prefrontal cortex reveals interdependence of neural activity during memoryguided saccades. J Neurophysiol 83:1550-1566.

Chafee MV, Crowe DA, Averbeck BB, Georgopoulos AP (2005) Neural correlates of spatial judgement during object construction in parietal cortex. Cereb Cortex 15:1393-1413.

Chafee MV, Averbeck BB, Crowe DA (2007) Representing spatial relationships in posterior parietal cortex: single neurons code object-referenced position. Cereb Cortex 17:2914-2932.

Crowe DA, Chafee MV, Averbeck BB, Georgopoulos AP (2004) Participation of primary motor cortical neurons in a distributed network during maze solution: representation of spatial parameters and time-course comparison with parietal area 7a. Exp Brain Res 158:28-34.

Crowe DA, Averbeck BB, Chafee MV (2008) Neural ensemble decoding reveals a correlate of viewer- to object-centered spatial transformation in monkey parietal cortex. J Neurosci 28:5218-5228.

Crowe DA, Averbeck BB, Chafee MV (2010) Rapid sequences of population activity patterns dynamically encode task-critical spatial information in parietal cortex. J Neurosci 30:11640-11653.

Freedman DJ, Assad JA (2006) Experience-dependent representation of visual categories in parietal cortex. Nature 443:85-88.

Freedman DJ, Miller EK (2008) Neural mechanisms of visual categorization: insights from neurophysiology. Neurosci Biobehav Rev 32:311-329.

Freedman DJ, Riesenhuber M, Poggio T, Miller EK (2001) Categorical representation of visual stimuli in the primate prefrontal cortex. Science 291:312-316.

Freedman DJ, Riesenhuber M, Poggio T, Miller EK (2002) Visual categorization and the primate prefrontal cortex: neurophysiology and behavior. J Neurophysiol 88:929-941.

Freedman DJ, Riesenhuber M, Poggio T, Miller EK (2003) A comparison of primate prefrontal and inferior temporal cortices during visual categorization. J Neurosci 23:5235-5246.

Genovesio A, Brasted PJ, Mitz AR, Wise SP (2005) Prefrontal cortex activity related to abstract response strategies. Neuron 47:307-320. 
Goldman-Rakic PS (1987) Circuitry of primate prefrontal cortex and regulation of behavior by representational memory. In: Handbook of physiology. The nervous system. Higher functions of the brain (Mountcastle VB, Plum F, Geiger SR, eds), pp 373-417. Bethesda, MD: American Physiological Society.

Johnson RA, Wichern DW (1998) Applied multivariate statistical analysis. Saddle River, NJ: Prentice Hall.

Kamigaki T, Fukushima T, Miyashita Y (2009) Cognitive set reconfiguration signaled by macaque posterior parietal neurons. Neuron 61:941-951.

Kamigaki T, Fukushima T, Miyashita Y (2011) Neuronal signal dynamics during preparation and execution for behavioral shifting in macaque posterior parietal cortex. J Cogn Neurosci 23:2503-2520.

Klecka WR (1980) Discriminant analysis. Newbury Park, CA: Sage Publications.

Mansouri FA, Matsumoto K, Tanaka K (2006) Prefrontal cell activities related to monkeys' success and failure in adapting to rule changes in a Wisconsin Card Sorting Test analog. J Neurosci 26:2745-2756.

Merchant H, Crowe DA, Robertson MS, Fortes AF, Georgopoulos AP (2011) Top-down spatial categorization signal from prefrontal to posterior parietal cortex in the primate. Front Syst Neurosci 5:69.

Miller EK (2000) The prefrontal cortex and cognitive control. Nat Rev Neurosci 1:59-65.

Miller EK, Freedman DJ, Wallis JD (2002) The prefrontal cortex: categories, concepts and cognition. Philos Trans R Soc Lond B Biol Sci 357:1123-1136.

Muhammad R, Wallis JD, Miller EK (2006) A comparison of abstract rules in the prefrontal cortex, premotor cortex, inferior temporal cortex, and striatum. J Cogn Neurosci 18:974-989.

Nakahara K, Hayashi T, Konishi S, Miyashita Y (2002) Functional MRI of macaque monkeys performing a cognitive set-shifting task. Science 295:1532-1536.

Nieder A, Miller EK (2004) A parieto-frontal network for visual numerical information in the monkey. Proc Natl Acad Sci U S A 101:7457-7462.

Romo R, Ruiz S, Crespo P, Zainos A, Merchant H (1993) Representation of tactile signals in primate supplementary motor area. J Neurophysiol 70:2690-2694.

Romo R, Merchant H, Zainos A, Hernández A (1997) Categorical perception of somesthetic stimuli: psychophysical measurements correlated with neuronal events in primate medial premotor cortex. Cereb Cortex 7:317-326.

Roy JE, Riesenhuber M, Poggio T, Miller EK (2010) Prefrontal cortex activity during flexible categorization. J Neurosci 30:8519-8528.

Stoet G, Snyder LH (2004) Single neurons in posterior parietal cortex of monkeys encode cognitive set. Neuron 42:1003-1012.

Stoet G, Snyder LH (2009) Neural correlates of executive control functions in the monkey. Trends Cogn Sci 13:228-234.

Swaminathan SK, Freedman DJ (2012) Preferential encoding of visual categorie in parietal cortex compared to prefrontal cortex. Nat Neurosci 15:315-320.

Wallis JD, Anderson KC, Miller EK (2001) Single neurons in prefrontal cortex encode abstract rules. Nature 411:953-956. 